\title{
Dust evolution across the Horsehead nebula
}

\author{
T. Schirmer ${ }^{1}$, A. Abergel ${ }^{1}$, L. Verstraete ${ }^{1}$, N. Ysard ${ }^{1}$, M. Juvela ${ }^{2}$, A. P. Jones ${ }^{1}$, and E. Habart ${ }^{1}$ \\ ${ }^{1}$ Université Paris-Saclay, CNRS, Institut d'astrophysique spatiale, 91405 Orsay, France \\ e-mail: thiebaut.schirmer@ias.u-psud.fr \\ ${ }^{2}$ Department of Physics, PO Box 64, University of Helsinki, 00014 Helsinki, Finland
}

Received 12 March 2020 / Accepted 30 April 2020

\begin{abstract}
Context. Micro-physical processes on interstellar dust surfaces are tightly connected to dust properties (i.e. dust composition, size, and shape) and play a key role in numerous phenomena in the interstellar medium (ISM). The large disparity in physical conditions (i.e. density and gas temperature) in the ISM triggers an evolution of dust properties. The analysis of how dust evolves with the physical conditions is a stepping stone towards a more thorough understanding of interstellar dust.

Aims. We highlight dust evolution in the Horsehead nebula photon-dominated region.

Methods. We used Spitzer/IRAC (3.6, 4.5, 5.8 and $8 \mu \mathrm{m})$ and Spitzer/MIPS (24 $\mu \mathrm{m})$ together with Herschel/PACS (70 and $160 \mu \mathrm{m})$ and Herschel/SPIRE (250, 350 and $500 \mu \mathrm{m})$ to map the spatial distribution of dust in the Horsehead nebula over the entire emission spectral range. We modelled dust emission and scattering using the THEMIS interstellar dust model together with the 3D radiative transfer code SOC.

Results. We find that the nano-grain dust-to-gas ratio in the irradiated outer part of the Horsehead is 6-10 times lower than in the diffuse ISM. The minimum size of these grains is 2-2.25 times larger than in the diffuse ISM, and the power-law exponent of their size distribution is 1.1-1.4 times lower than in the diffuse ISM. In the denser part of the Horsehead nebula, it is necessary to use evolved grains (i.e. aggregates, with or without an ice mantle).

Conclusions. It is not possible to explain the observations using grains from the diffuse medium. We therefore propose the following scenario to explain our results. In the outer part of the Horsehead nebula, all the nano-grain have not yet had time to re-form completely through photo-fragmentation of aggregates and the smallest of the nano-grain that are sensitive to the radiation field are photo-destroyed. In the inner part of the Horsehead nebula, grains most likely consist of multi-compositional mantled aggregates.
\end{abstract}

Key words. ISM: individual objects: Horsehead nebula - photon-dominated region - dust, extinction - evolution

\section{Introduction}

Interstellar dust plays an essential role within the interstellar medium (ISM) through different microphysical processes that occur on dust surfaces that can heat the gas, such as the photoelectric effect (e.g. Bakes \& Tielens 1994; Weingartner \& Draine 2001a), or cool the gas through gas-grain collisions (Burke \& Hollenbach 1983). By acting as a catalyst, allowing atoms and molecules to react on its surface, dust is strongly involved in the chemistry of the ISM (e.g. Hollenbach \& Salpeter 1971; Bron et al. 2014; Jones \& Habart 2015; Wakelam et al. 2017). Dust also plays a role in the redistribution of UV-visible stellar radiation into IR-millimeter radiation, a process that depends on the dust mass and the volume of dust grains (e.g. Draine 2003; Compiègne et al. 2011). The efficiency of these processes strongly depends on dust properties such as the grain size, composition and shape. It is therefore important to constrain dust properties in order to understand the different phenomena that take place in the ISM. To this purpose, several dust models have been developed and are classified into three categories: dust composed of silicate and of graphite (e.g Mathis et al. 1977; Draine \& Lee 1984; Kim et al. 1994), and an extension of these models that uses polycyclic aromatic hydrocarbons (PAHs; e.g. Siebenmorgen \& Kruegel 1992; Li \& Draine 2001; Weingartner $\&$ Draine 2001b). As a result of fragmentation and coagulation processes in the ISM, dust models with grains that have a core-mantle structure (e.g. Desert et al. 1990; Jones et al. 1990; Li \& Greenberg 1997) and dust composite models composed of silicate and carbon grain aggregates (e.g. Mathis \& Whiffen 1989; Zubko et al. 2004) have been proposed. In this paper, we use the THEMIS dust model (see Jones et al. 2013, 2014, 2017; Köhler et al. 2014, 2015; Ysard et al. 2015), which was developed in combination with the results of laboratory experiments and astronomical observations. The cornerstone of this model is its self-consistent view of the evolution of the dust constituents through the ISM. This view is required for understanding dust evolution in response to the local ISM conditions (i.e. density and radiation field).

Some of the first evidence of dust evolution was shown by Fitzpatrick \& Massa (1986) through the variation in the $2175 \AA$ interstellar bump from diffuse $\left(R_{V}=3.1\right)$ to denser regions (up to $R_{V} \sim 5.5$ ). Similarly, other studies (e.g. Cardelli et al. 1989; Cardelli \& Clayton 1991; Campeggio et al. 2007) found the same variations, which were for the first time explained by Kim et al. (1994) by stating that these observations are consistent with a decrease in the carbonaceous nano-grain abundance (relative to the gas) together with an increase in larger grain abundance. It is also possible to follow dust evolution from its emission in the mid-IR (due to stochastically heated nano-grain) and in the far-IR (where large grains at thermal equilibrium emit). This has led to a wealth of studies (e.g. Boulanger et al. 1990; Laureijs et al. 1991; Abergel et al. 1994; Bernard et al. 1999; Stepnik et al. 2003; Flagey et al. 2009) revealing that nanograin disappear in dense regions as they coagulate onto larger grains. Dust evolution is also highlighted by variation of its 
far-IR opacity with the local environment (e.g. Juvela et al. 2011, 2015; Planck Collaboration XXIV 2011; Martin et al. 2012; Roy et al. 2013; Ysard et al. 2013; Köhler et al. 2015), which has been explained with dust coagulation and accretion of ice mantles. This scenario is supported by numerical simulations of dust evolution in dense regions (e.g. Ossenkopf \& Henning 1994; Ormel et al. 2011; Köhler et al. 2015).

Photon-dominated regions (PDRs; Hollenbach \& Tielens 1997, 1999) correspond to the interface between HII regions and molecular clouds that are irradiated by energetic stars that are located close by. In these regions, the physical conditions vary widely, and hence PDRs are a unique place to study how do dust, gas, and local physical conditions evolve with depth. Based on dust emission variations in the mid-IR observed with Spitzer in several PDRs (Ced 201, NGC 7023, and the western filament of $\rho$ Ophiuchi), Berné et al. (2007) construed that such variations can be explained by the photo-processing of carbonaceous nanograin, a scenario that was later reinforced in other PDRs (Abergel et al. 2010; Pilleri et al. 2012, 2015; Boersma et al. 2014). Using far-IR observations from Herschel together with the near- and mid-IR observations from Spitzer, Arab et al. (2012) found that the carbonaceous nano-grain abundance decreases together with an increase in the opacity of the large grains in the Orion bar. They claimed that these variations are likely due to coagulation processes in the denser part of this region. Evidence of dust evolution has also been shown in IC 63 based on extinction mapping (Van De Putte et al. 2019).

In this paper, we focus on a well-known PDR, the Horsehead nebula (Horsehead for short), which has previously been studied from the perspective of dust observations (e.g. Abergel et al. 2003; Teyssier et al. 2004; Compiègne et al. 2007, 2008; Pety et al. 2005; Arab 2012), gas observations (e.g. Habart et al. 2005; Goicoechea et al. 2006; Gerin et al. 2009; Guzmán et al. 2011; Pety et al. 2012; Le Gal et al. 2017) and laboratory experiments (Alata et al. 2015). The most important question we try to answer is the way in which the dust properties change with physical conditions. We investigate whether it is possible to understand these observations with grains from the diffuse ISM. Alternatively, there might be a viable dust evolution scenario that can explain the observations and is consistent with the physical conditions in the Horsehead.

The paper is organised as follows. In Sect. 2 we describe previous studies and the observations of the Horsehead. In Sect. 3 we detail the dust model we use, THEMIS, as well as the local dust emission tool, DustEM. We also present the effects of variations in dust properties on its emission in the optically thin case with DustEM in order to distinguish variations in the dust spectrum that are due to changes in dust properties and those that are due to radiative transfer effects. In Sect. 4 we present SOC, the $3 \mathrm{D}$ radiative transfer code we use, as well as the effect of variations in the dust parameters on dust emission in the optically thick case, in the case of the Horsehead. In Sect. 5 we compare our model with the observations and present the best parameters we obtain. In Sect. 6 we discuss our results and propose a scenario of dust evolution in the Horsehead. Finally, we present our conclusions in Sect. 7.

\section{Prototypical PDR: the Horsehead nebula}

As physical conditions vary widely and are spatially resolved in nearby PDRs, they are the ideal place to study dust evolution as a function of physical conditions. First, we introduce the different studies that have been made of the Horsehead; second, we present the observations of the Horsehead obtained with Spitzer and Herschel; and third, we describe the density profile that we used to perform radiative transfer across the Horsehead.

\subsection{Well studied PDR}

The Horsehead is an archetypal PDR situated at $\sim 400 \mathrm{pc}$ (Anthony-Twarog 1982) that is illuminated by the binary star $\sigma$-Orionis which is an O9.5V binary system (Warren \& Hesser 1977 ) with an effective temperature of $T_{\text {eff }} \sim 34600 \mathrm{~K}$ (Schaerer $\&$ de Koter 1997) located at a projected distance $d_{\text {edge }} \sim 3.5 \mathrm{pc}$ from the Horsehead edge. Observations of the Horsehead have been made in the visible (e.g. de Boer 1983; Neckel \& Sarcander 1985) and at millimeter wavelengths for ${ }^{12} \mathrm{CO}$ and ${ }^{13} \mathrm{CO}$ (e.g. Milman et al. 1975), CS (e.g. Lada et al. 1991), $C^{+}$(e.g. Zhou et al. 1993) and ${ }^{13}$ CO (e.g. Kramer et al. 1996).

Later, mid-IR observations (Abergel et al. 2003) with ISOCAM highlighted that the Horsehead is likely to be seen edge-on and therefore offers us a unique opportunity to study dust, gas and the evolution of local physical conditions with depth into the Horsehead. This has led to many studies at millimeter wavelengths for $\mathrm{CO}$ (Pound et al. 2003), $\mathrm{C}^{18} \mathrm{O}$ (Hily-Blant et al. 2005), CS, $\mathrm{C}^{34} \mathrm{~S}$ and $\mathrm{HCS}^{+}$(Goicoechea et al. 2006), CI and CO (Philipp et al. 2006), DCO ${ }^{+}$(Pety et al. 2007), $\mathrm{HCO}$ and $\mathrm{H}^{13} \mathrm{CO}^{+}$ (Gerin et al. 2009), $\mathrm{H}^{13} \mathrm{CO}^{+}, \mathrm{DCO}^{+}$, and $\mathrm{HCO}^{+}$(Goicoechea et al. 2009), $\mathrm{H}_{2} \mathrm{CO}$ (Guzmán et al. 2011), $\mathrm{CF}^{+}$(Guzmán et al. 2012), $1-\mathrm{C}_{3} \mathrm{H}^{+}$(Pety et al. 2012), $\mathrm{CH}_{3} \mathrm{CN}, \mathrm{HC}_{3} \mathrm{~N}$, and $\mathrm{C}_{3} \mathrm{~N}$ (Gratier et al. 2013), $\mathrm{H}_{2} \mathrm{CO}$ and $\mathrm{CH}_{3} \mathrm{OH}$ (Guzmán et al. 2013), and $\mathrm{HCOOH}, \mathrm{CH}_{2} \mathrm{CO}, \mathrm{CH}_{3} \mathrm{CHO}$ and $\mathrm{CH}_{3} \mathrm{CCH}$ (Le Gal et al. 2017).

Teyssier et al. (2004) found for the dust that although small hydrocarbons are expected to be photo-destroyed by the intense UV field at the edge of the Horsehead, they still exist. The authors suggest that the photo-erosion of carbonaceous nanograin into small hydrocarbons is more efficient than the photodestruction of small hydrocarbons at the Horsehead edge. This scenario is reinforced by observations in Pety et al. (2005), who found hydrocarbons such as $\mathrm{CCH}, \mathrm{c}-\mathrm{C}_{3} \mathrm{H}_{2}$, and $\mathrm{C}_{4} \mathrm{H}$ in the UVirradiated outer part of the Horsehead. It is also supported by laboratory experiments on thermal processed and UV-irradiated dust grains analogues (see Smith 1984; Zubko et al. 2004; Alata et al. 2014, 2015; Duley et al. 2015). Based on Spitzer observations, Compiègne et al. (2007) proposed a scenario where PAHs survive in HII regions, and Compiègne et al. (2008) reported that spectral variations in the mid-IR cannot be explained by radiative transfer effects alone and therefore are a consequence of the dust evolution across the Horsehead.

\subsection{Observations with Spitzer and Herschel}

We used Spitzer and Herschel observations (see Appendix B) of the Horsehead in ten photometric bands from 3.6 to $500 \mu \mathrm{m}$, which cover almost the entire dust spectrum. The processing of the Spitzer maps is detailed in Bowler et al. (2009). Data were processed in the HIPE environment, with standard Herschel corrections for instrumental effects and glitches. PACS $70 \mu \mathrm{m}$ and $160 \mu \mathrm{m}$ maps were obtained after the superposition of two observations with a scan speed of $20^{\prime \prime} \mathrm{s}^{-1}$, whose directions were perpendicular to one another. The overall duration of these observations is $4122 \mathrm{~s}$, and they cover $8.8^{\prime} \times 4.5^{\prime}$ of the Horsehead. In SPIRE 250, 350 and $500 \mu \mathrm{m}$, they were obtained after the superposition of two observations with a scan speed of $30^{\prime \prime} \mathrm{s}^{-1}$, whose directions were perpendicular to one other. The overall duration of these observations is $1341 \mathrm{~s}$, and they cover $8^{\prime} \times 8^{\prime}$ of 

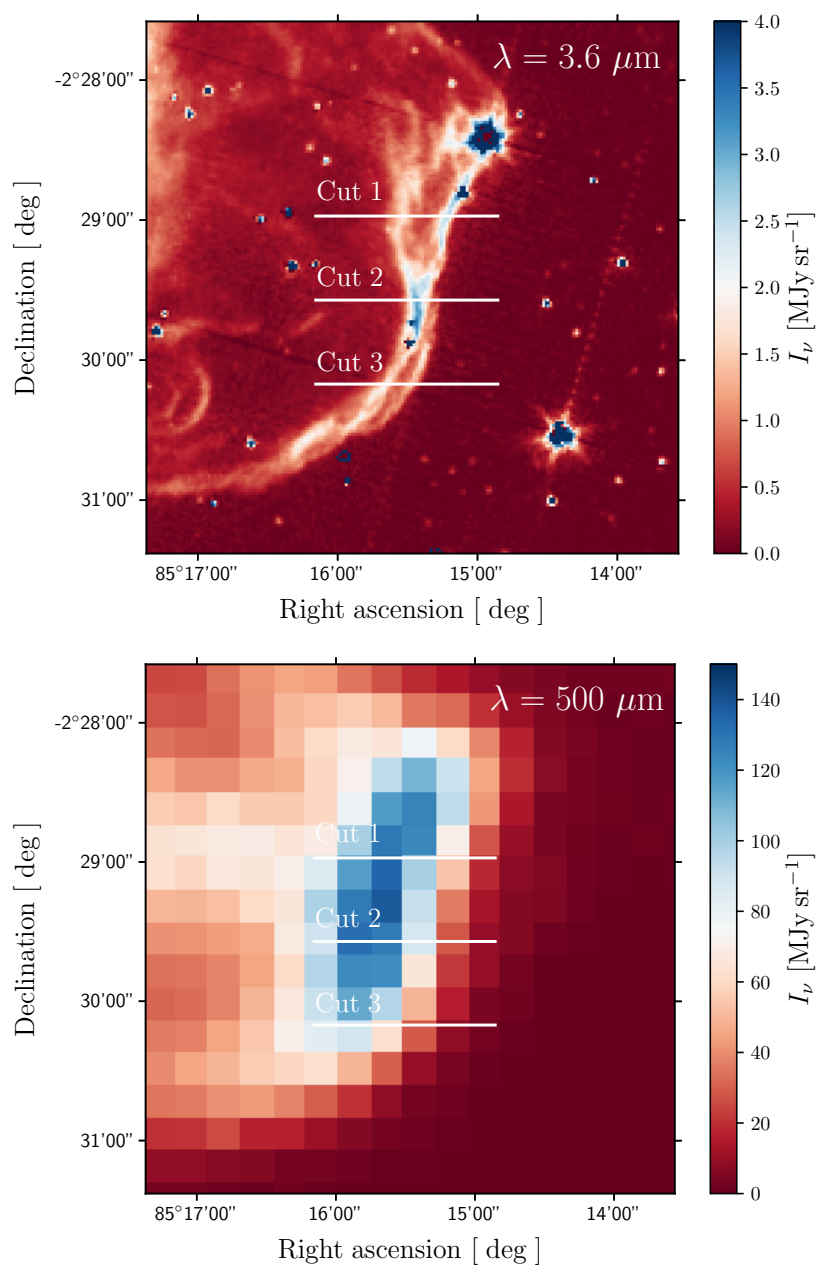

Fig. 1. Top: Horsehead seen with IRAC at $3.6 \mu \mathrm{m}$. The three white solid lines correspond to the three cuts we used in our study. Bottom: Horsehead seen with SPIRE at $500 \mu \mathrm{m}$.

the Horsehead. Striping induced by offsets in the flux calibration from one detector to another was removed using the Scan Map Destriper module included in the HIPE environment.

We studied the observed emission profiles through three different cuts across the Horsehead (see Fig. 1). The calibration uncertainty in the IRAC bands $\left(\mathrm{IRAC}_{3.6}, \mathrm{IRAC}_{4.5}, \mathrm{IRAC}_{5.8}\right.$, and IRAC $_{8.0}$ ) is $2 \%$ (Reach et al. 2005), $4 \%$ in MIPS $_{24}$ (Engelbracht et al. 2007), 5\% in $\mathrm{PACS}_{70}$ (Gordon et al. 2007), 12\% in PACS $_{160}$ (Stansberry et al. 2007), and $15 \%$ in the three SPIRE bands (Swinyard et al. 2010). We considered all these errors to be independent of the wavelength to first order. We also considered that the emission in all of these ten bands comes from dust, which is not completely the case in IRAC $_{3.6}$ and IRAC 4.5 . We estimated with a model of atomic and molecular gas in PDRs, the Meudon PDR Code (Le Petit et al. 2006), that gas can contribute less than $10 \%$ of the flux. However, this contribution does not affect the bulk of our results, therefore we consider that the observed emission is dust emission. Nevertheless, we must be careful in interpreting the observations because gas emission can be higher than dust emission in photometric bands that cover shorter wavelengths.

\subsection{Density profile across the Horsehead}

We performed radiative transfer calculations, which require information on the density profile across the Horsehead. We

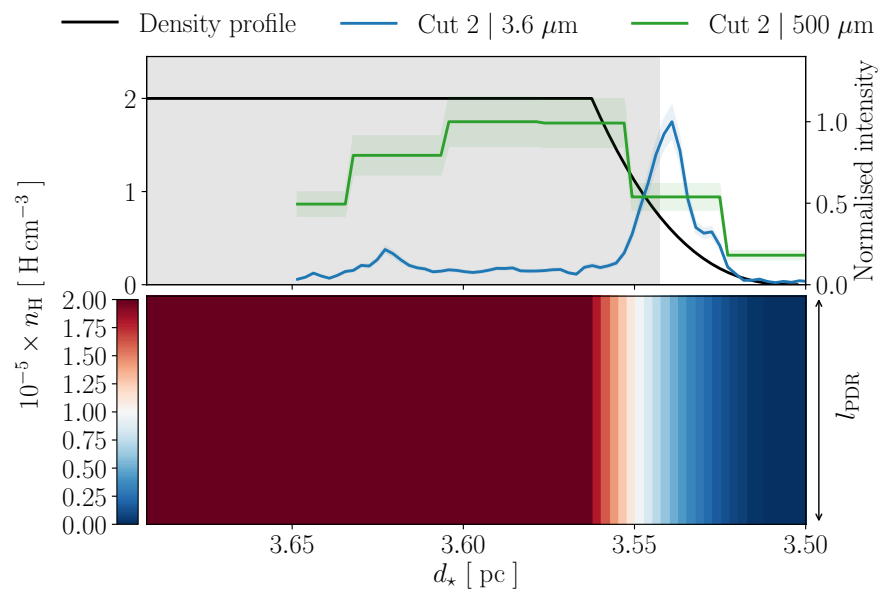

Fig. 2. Top: assumed density profile across the Horsehead (black line, see Sect. 2.3). Normalised dust-observed emission (blue line) in $\mathrm{IRAC}_{3.6}$ (see Fig. 1). Normalised dust observed emission (green line) in SPIRE $_{500}$. The grey part corresponds to the inner part of the Horsehead, defined in Sect. 4.4. Bottom: density profile in 2D-space defined by the distance to the star, $d_{\star}$, and the length of the Horsehead along the line of sight, $l_{\mathrm{PDR}}$.

used the profile described in Habart et al. (2005). Because the $\mathrm{H}_{2}$ 1-0 S(1) fluorescent emission is very sensitive to both the radiation field and the gas density, they observed this line with the SOFI instrument at the NTT. This observation was combined with previous observations of $\mathrm{H}_{\alpha}$ and dust mid-IR emission in order to constrain the density profile at the edge of the Horsehead. Habart et al. (2005) also used CO millimeter observations from the IRAM 30 m telescope (Abergel et al. 2003; Teyssier et al. 2004) and the Plateau de Bure Interferometer (Pety et al. 2005), as well as $1.2 \mathrm{~mm}$ dust continuum emission obtained with MAMBO at the IRAM $30 \mathrm{~m}$ telescope (Teyssier et al. 2004) to constrain the density profile in the inner part. All these observations were interpreted with the Meudon PDR code. This density profile (see Fig. 2, upper panel) was also used in Compiègne et al. (2008) and Arab (2012) and is defined as follows:

$n_{\mathrm{H}}(z)= \begin{cases}n_{0} \times\left(\frac{z}{z_{0}}\right)^{\gamma} & \text { if } z<z_{0} \\ n_{0} & \text { if } z>z_{0},\end{cases}$

where

$n_{0}=2 \times 10^{5} \mathrm{Hcm}^{-3} ; z_{0}=0.06 \mathrm{pc} ; \gamma=2.5 ; z=d_{\star}-d_{\text {edge }}$,

with $z$ the position from the edge of the Horsehead, $\gamma$ the powerlaw exponent of the gas density profile and $z_{0}$ the depth beyond which constant density $n_{0}$ is reached.

The authors also estimated the length of the Horsehead along the line of sight, $l_{\mathrm{PDR}}$. They found that this parameter is constrained to be between 0.1 and $0.5 \mathrm{pc}$. We assumed that the density profile is independent of the position along the line of sight (see Fig. 2, bottom panel).

\section{Dust modelling}

The interpretation of the multi-wavelength observations of the Horsehead depends on its structure, the incident radiation field, and the dust model. We therefore need a dust model and modelling tools to compute dust emission based on the local physical conditions. First, we describe our adopted dust model THEMIS; 
second, we introduce DustEM, which we used to compute the local dust emission, and we describe the evolution of dust emission with its properties in the optically thin case using DustEM.

\subsection{THEMIS}

The Heterogeneous dust Evolution Model for Interstellar Solids, THEMIS $^{1}$ (e.g., Jones et al. 2013, 2017; Köhler et al. 2014) is based on observational constraints and laboratory measurements on interstellar dust analogues that are amorphous hydrocarbons, a-C(:H) (e.g., Jones 2012a,b,c), and amorphous silicates, a-Sil. This model includes dust evolution through processes such as photo-processing, fragmentation, and coagulation resulting from wide variations in the physical condition of the ISM.

THEMIS for the diffuse ISM (Jones et al. 2013; Köhler et al. 2014; Ysard et al. 2015) is composed of amorphous silicates (aSil/a-C) surrounded by a mantle of aromatic-rich carbon, and amorphous hydrocarbon solids that encompass a-C:H material that is $\mathrm{H}$-rich and therefore aliphatic-rich, and a-C material that is $\mathrm{H}$-poor and therefore aromatic-rich. When the typical penetration depth of a UV photon in an amorphous carbon grain is about $20 \mathrm{~nm}$ (see Fig. 15 in Jones 2012b), carbonaceous grains that are smaller than $20 \mathrm{~nm}$ are entirely photo-processed and therefore aromatic. Larger grains are composed of an aliphatic core and surrounded by an aromatic mantle that is $20 \mathrm{~nm}$ thick, which prevents photo-processing of the core and therefore allows the core to remain aliphatic. This view provides us with a continuous description of carbonaceous grains from the smallest that mostly contain aromatic cycles and are stochastically heated to the largest that are at thermal equilibrium. Details about the size distribution are listed in Table A.1. Because these grains are composed of either an a-C:H core or a silicate core surrounded in both cases by an aromatic carbonaceous mantle, they are called core-mantle grains $(\mathrm{CM})$.

In the dust evolution framework assumed by THEMIS (Jones et al. 2014), large grains can form a second mantle either through accretion of $\mathrm{C}$ and $\mathrm{H}$ atoms, available in the gas phase or through coagulation of a-C nano-grains on the surfaces of larger grains. These grains are called core-mantle-mantle (CMM) grains. In denser regions, CMM grains coagulate together to form aggregates (Köhler et al. 2015) called aggregate-mantle-mantle (AMM) grains. Where the shielding from energetic photons is efficient enough, a mantle of water ice can form around AMM grains, leading to aggregated-mantle-mantle-ice (AMMI) grains.

In the following, we use several dust mixtures (see Fig. 1 in Jones et al. 2017). Parameters associated with the size distribution of these dust mixtures are listed in Table A.1 and the size distributions are shown in Fig. 3 (upper panel) with the associated spectra (see Fig. 3, bottom panel) computed with DustEM (see Sect. 3.2) using a radiation field corresponding to a star at $34600 \mathrm{~K}$ with $G_{0}=100$, where

$G_{0}=\frac{1}{1.6 \times 10^{-3}\left(\mathrm{erg} \mathrm{s}^{-1} \mathrm{~cm}^{-2}\right)} \int_{6 \mathrm{eV}}^{13.6 \mathrm{eV}} I_{\nu} \mathrm{d} v$.

In the near-IR (1-5 $\mu \mathrm{m})$ and mid-IR $(5-30 \mu \mathrm{m})$, dust emission comes mainly from the a-C grains. In the far-IR (from 50 to $500 \mu \mathrm{m}$ ), dust emission comes mainly from a-Sil/a-C and a-C:H/a-C grains.
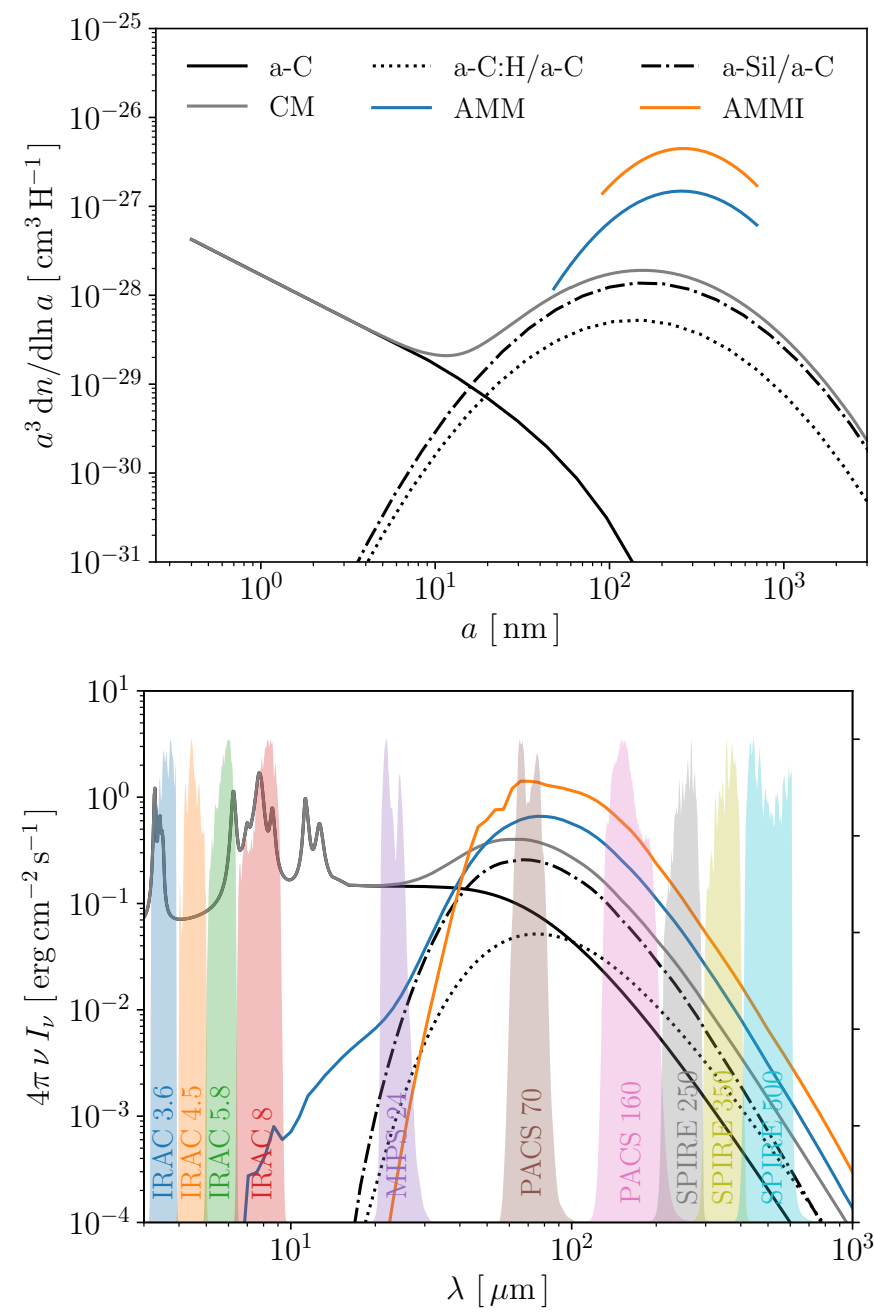

Fig. 3. Top: size distributions of the dust mixtures from THEMIS (parameters are listed in Table A.1) for CM-grains, i.e. diffuse ISMlike dust (grey line), AMM (blue line), and AMMI (orange line), i.e. grain aggregates typical of starless dense clouds. The black line, the dotted line, and the dash-dotted line correspond to a-C, a-C:H/a-C, and a-Sil/a-C respectively, which are the sub-components of the $\mathrm{CM}$ grains. Bottom: associated spectra, computed with DustEM with a radiation field corresponding to a star at $34600 \mathrm{~K}$ with $G_{0}=100$.

\subsection{Influence of dust properties on dust emission with DustEM}

DustEM $^{2}$ (Compiègne et al. 2011) is a modelling tool that computes the extinction, the emission, and the polarisation of interstellar dust grains heated by photons in the optically thin case (i.e. no radiative transfer).

In order to distinguish the effects of radiative transfer from variations in the dust properties on emission, we studied the influence of such variations with DustEM. We modified the following parameters:

1. The a-C abundance, i.e. the a-C mass to gas ratio, $M_{\mathrm{a}-\mathrm{C}} / M_{\mathrm{H}}$, which we varied from $0.01 \times 10^{-2}$ to $0.20 \times 10^{-2}$ in steps of $0.01 \times 10^{-2}$.

2. The a-C minimum size, $a_{\min , \mathrm{a}-\mathrm{C}}$, which we varied from $0.4 \mathrm{~nm}$ to $0.9 \mathrm{~nm}$ in steps of $0.02 \mathrm{~nm}$.

3. The slope of the a-C power-law size distribution, $\alpha$, which we varied from -6 to -4 in steps of 0.1 .

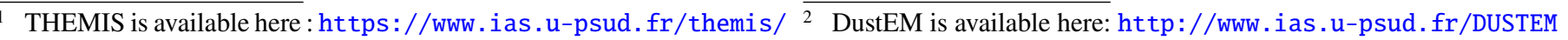



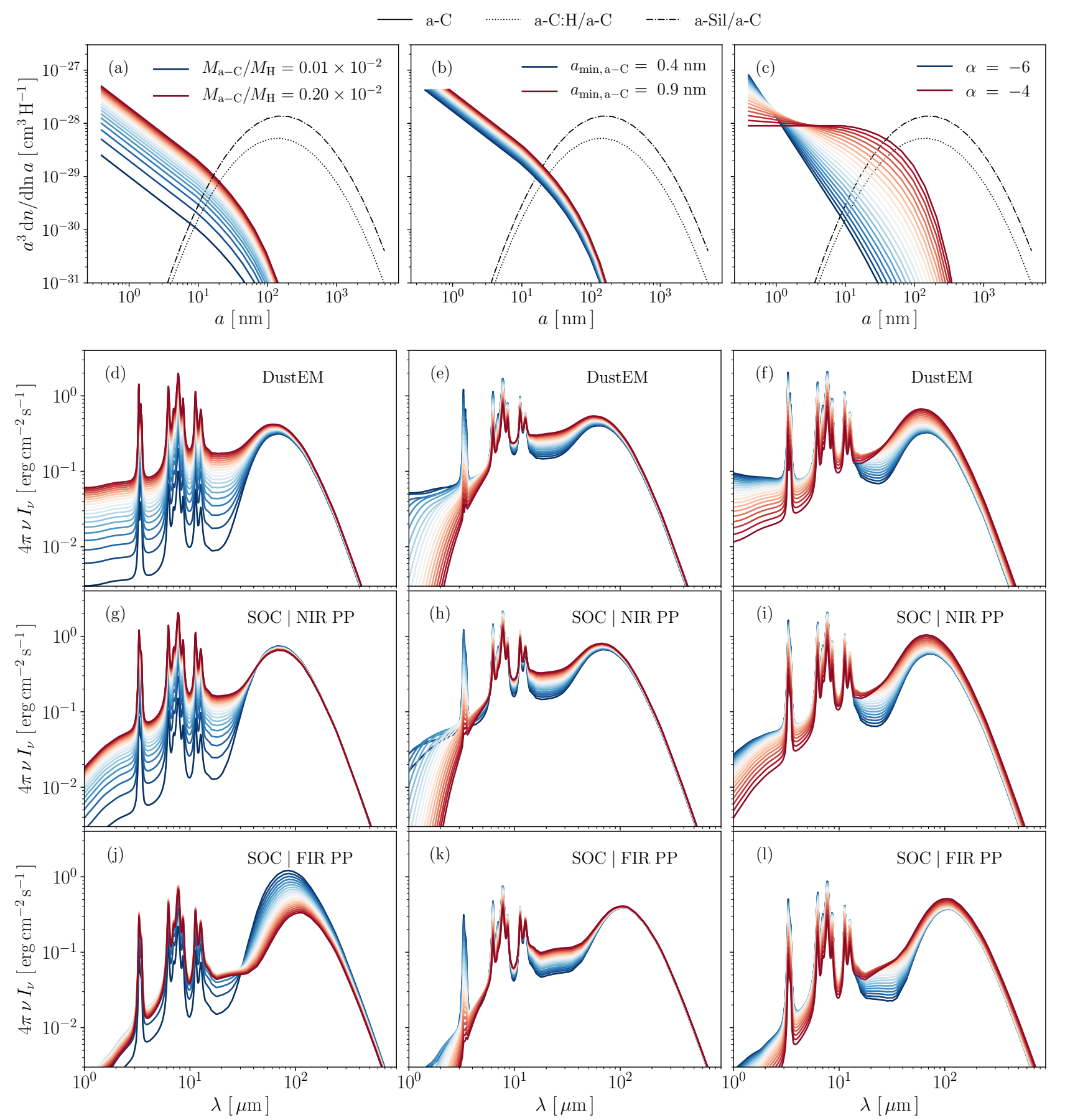

Fig. 4. Dust size distributions for different values of $M_{\mathrm{a}-\mathrm{C}} / M_{\mathrm{H}}$ (panel $a$ ), $a_{\min , \mathrm{a}-\mathrm{C}}($ panel $b$ ) and $\alpha$ (panel $c$ ). Panels $d-l$ : dust spectra for the size distributions shown in the corresponding top-row panels. The spectra are computed with DustEM (panels $d-f$ ) and with radiative transfer for the near-IR (panels $g-i$ ) and the far-IR (panels $j-l$ ) peak positions.

The results are shown in Fig. 4, where the spectra in panels d, $\mathrm{e}$, and $\mathrm{f}$ are associated with the size distributions in panels $\mathrm{a}, \mathrm{b}$, and $\mathrm{c}$, respectively. All the spectra are obtained with a radiation field that corresponds to a blackbody at $34600 \mathrm{~K}$ scaled so that $G_{0}=100$ (i.e. the radiation field illuminating the Horsehead).

A decrease in $M_{\mathrm{a}-\mathrm{C}} / M_{\mathrm{H}}$ or an increase in $a_{\text {min, a-C }}$ or $\alpha$ leads to a decrease in the smallest a-C grains and accordingly to a decrease in the near-IR emission. Because the total dust mass is fixed, an increase in $a_{\text {min, a-C }}$ or $\alpha$ leads to a redistribution of the dust mass from the smallest to the largest a- $\mathrm{C}$ grains and therefore to an increase in the mid-IR emission. In the far-IR, dust emission is unaffected by variations in $M_{\mathrm{a}-\mathrm{C}} / M_{\mathrm{H}}, a_{\mathrm{min}, \mathrm{a}-\mathrm{C}}$, and $\alpha$ because a-C grains are barely responsible for any dust emission at these long wavelengths. However, the dust emission in the far-IR slightly increases with an increase in $\alpha$ as the mass of the largest a-C increases significantly, unlike an increase in $a_{\text {min, a-C. }}$.

\section{Radiative transfer modelling within the Horsehead}

The Horsehead is an optically thick region that requires radiative transfer modelling to properly interpret our multi-wavelength 
observations. We present the 3D radiative transfer code SOC that we used here. Performing radiative transfer is time consuming, and we here therefore explore the influence of the Horsehead length along the line of sight $l_{\mathrm{PDR}}$, and dust properties (i.e. $\left.M_{\mathrm{a}-\mathrm{C}} / M_{\mathrm{H}}, a_{\text {min, a-C }}, \alpha\right)$ on dust emission after radiative transfer calculations.

\subsection{Radiative transfer code : SOC}

SOC is a 3D Monte Carlo radiative transfer code, parallelised using OpenCL libraries (Juvela 2019). It computes dust emission and scattering. SOC has been benchmarked with other radiative transfer codes in Gordon et al. (2017) and used in Juvela et al. (2018a,b, 2019).

The radiation field corresponds to that of a blackbody at $34600 \mathrm{~K}$ produced by a star to which a dilution factor has been applied to obtain $G_{0}=100$ at the Horsehead edge. This radiation field is estimated on a logarithmic grid of 334 frequencies that extends from $3 \times 10^{9} \mathrm{~Hz}$ to $3 \times 10^{16} \mathrm{~Hz}$. Because the Horsehead edge is located outside the HII region, there are no photons above $13.6 \mathrm{eV}$, hence we applied the Lyman cut to the radiation field that heats the Horsehead edge. Each frequency was simulated with $10^{6}$ photons.

In SOC, clouds can be defined on regular Cartesian grids or octree grids. We modelled the Horsehead using a Cartesian grid that contains $N_{X} \times N_{Y} \times N_{Z}$ cubes that measure 0.0025 pc per side. $N_{X}$ is equal to 77 and corresponds to the number of cubes along the Horsehead-star axis. $N_{Y}$ is equal to 7 and corresponds to the number of cubes along the axis perpendicular to the Horsehead-star axis and the line-of-sight axis (i.e. the observer-Horsehead axis). $N_{Z}$ corresponds to the number of cubes in the Horsehead along the line of sight and therefore depends on the value of $l_{\mathrm{PDR}}: N_{Z}=l_{\mathrm{PDR}} / 0.0025 \mathrm{pc}$. For each cube, we associated a value of the gas density as described in Sect. 2.3.

We computed only dust emission because regardless of the dust properties, dust scattering contributes less than $1 \%$ to the total dust brightness in the near-IR photometric bands. After the integration along the line of sight, dust emission profiles across the Horsehead were integrated into the different photometric bands and convolved with the PSFs.

\subsection{Influence of $I_{P D R}$ on dust emission}

In the following, we study dust emission at two positions : the near-IR peak position (NIR PP) in the Horsehead, and the far-IR peak position (FIR PP). These positions corresponds to the peak of emission in IRAC $_{3.6}$ and SPIRE 500 , respectively, shown in the Fig. 2. In order to facilitate reading the results we obtained, we also introduce $I_{\bmod , \max }(i)=\max \left(I_{\bmod , i}(z)\right)$ where $I_{\bmod , i}(z)$ is the dust-modelled emission in the $i$ th band at the position $z$ along the cut.

Whether it is at the NIR PP or at the FIR PP, dust emission increases in all bands with $l_{\mathrm{PDR}}$ (see Fig. 5, top and middle panels) because the dust mass increases along the line of sight as the column density ${ }^{3}$ increases with $l_{\mathrm{PDR}}$. Moreover, the dust emission increases linearly with $l_{\mathrm{PDR}}$ (see Fig. 5, bottom panel) revealing that dust self-absorption, which depends on both the column density and on the wavelength, is negligible at these wavelengths in the $l_{\mathrm{PDR}}$ range we considered. Consequently, we considered that the intensity increases linearly with $l_{\mathrm{PDR}}$ in the near-, mid-, and far-IR and does not affect the shape of the

\footnotetext{
$3 N_{\mathrm{H}}(z)=n_{\mathrm{H}}(z) l_{\mathrm{PDR}}$
}
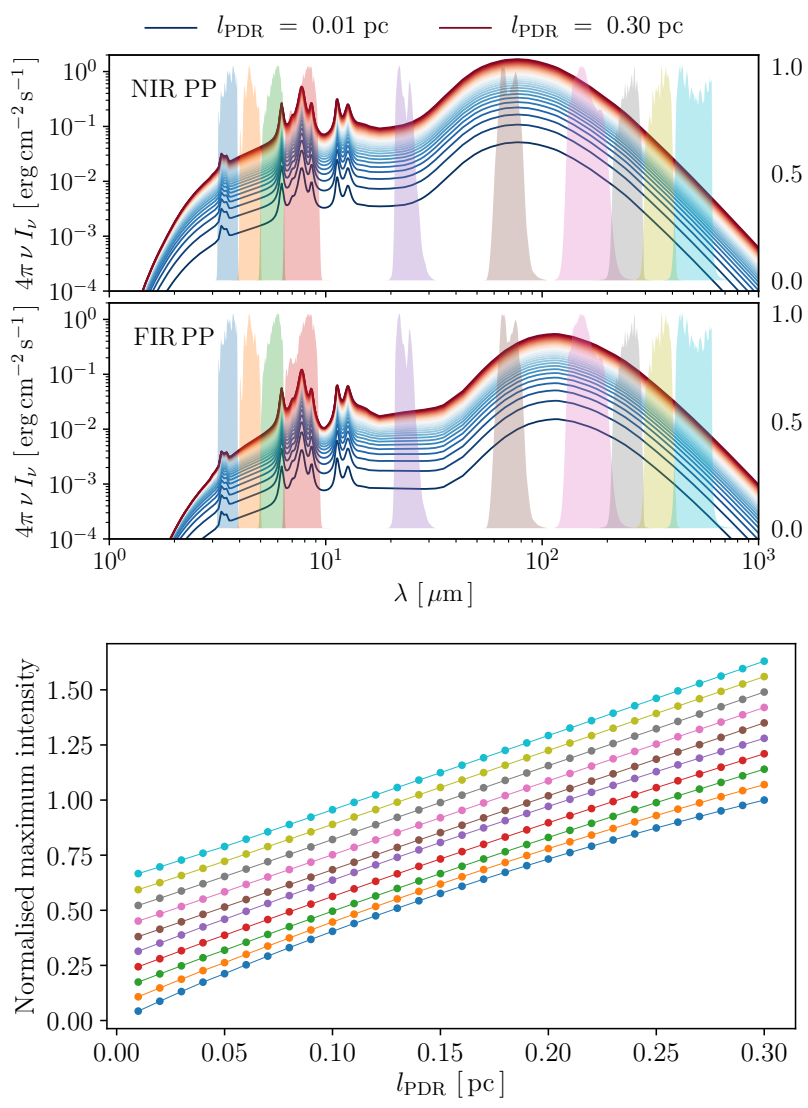

Fig. 5. Top: dust-modelled spectra with $\mathrm{SOC}$ and using CM grains, i.e. diffuse ISM-like dust, at the near-IR peak position (NIR PP) for $l_{\mathrm{PDR}}$ varying from $0.01 \mathrm{pc}$ (blue line) to $0.30 \mathrm{pc}$ (red line) with a step of $0.01 \mathrm{pc}$. The ten photometric bands are shown in colours behind the lines. Middle: same at the far-IR peak position (FIR PP). Bottom: normalised $I_{\mathrm{mod}, \max }(i)$ for each band as a function of $l_{\mathrm{PDR}}$. Colours refer to the different photometric bands shown in the upper panels. Lines are shifted for clarification (from top to bottom in the order of decreasing wavelength).

dust spectrum. In the following, we therefore consider $l_{\mathrm{PDR}}$ as a multiplying factor on the dust spectrum.

\subsection{Influence of dust properties on dust emission after radiative transfer}

In contrast to Sect. 3.2, where we studied the influence of dust properties on dust emission in the optically thin case, we study here the influence of these properties in the optically thick case by performing a radiative transfer calculation. These results are shown in Fig. 4, where the spectra in panels $\mathrm{g} / \mathrm{j}, \mathrm{h} / \mathrm{k}$, and $\mathrm{i} / \mathrm{l}$ are associated with the size distributions in panel a, b, and c, respectively. Spectra in panels $g, h$ and $i$ are located at the NIR PP, and those in panels j, k, and 1 are located at the FIR PP.

Dust grains are warmer at the NIR PP (see Fig. 4, panels g, h and i) than at the FIR PP (panels $\mathrm{j}, \mathrm{k}$ and $\mathrm{l}$ ) because the maximum intensity shifts towards longer wavelengths. This effect is due to the damping of the radiation field with increasing depth into the Horsehead.

At the NIR PP, dust emission in the far-IR does not vary with $M_{\mathrm{a}-\mathrm{C}} / M_{\mathrm{H}}$ (see Fig. 4, panel g), in contrast to what we show in the inner part (panel j). Because dust emission in the far-IR is unaffected by variations in $M_{\mathrm{a}-\mathrm{C}} / M_{\mathrm{H}}$ in the optically thin case (see Sect. 3.2), this is strictly a radiative transfer effect. Because the a-C grains bear a large fraction of the total dust cross-section, 

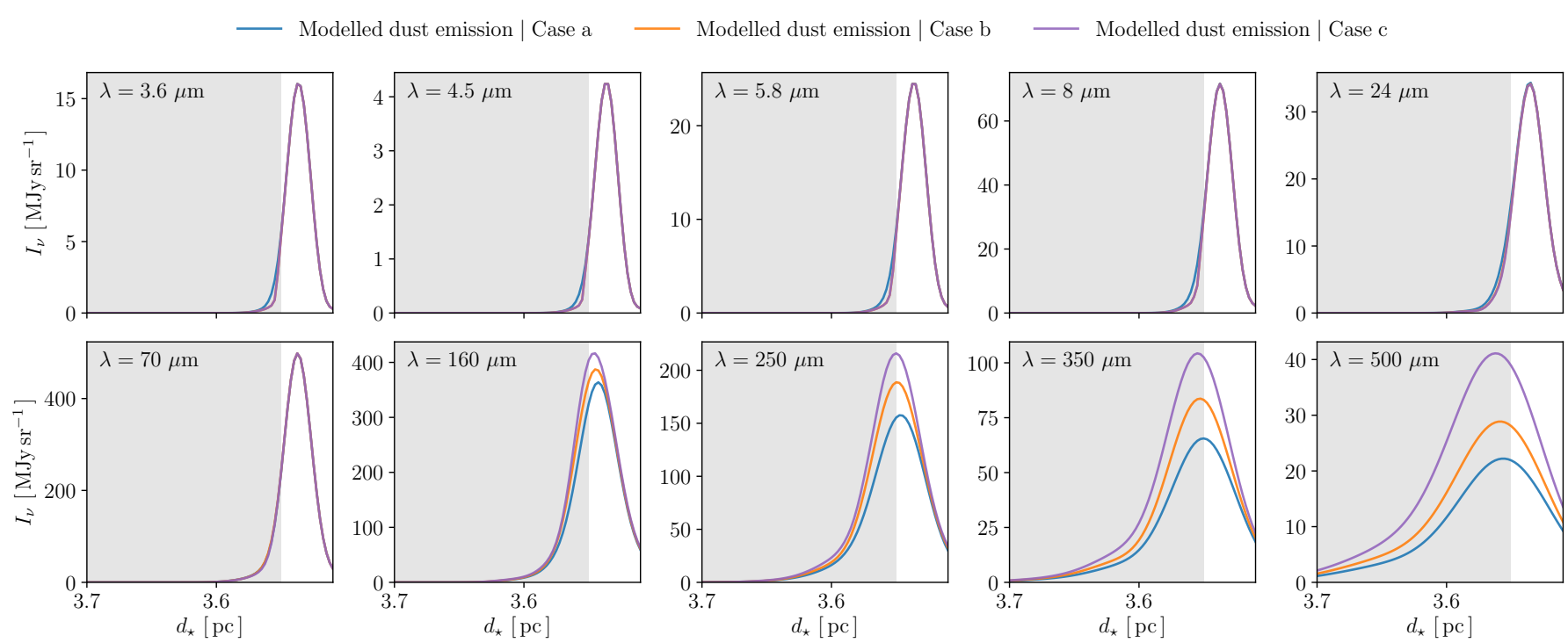

Fig. 6. Dust-modelled emission profiles in the ten photometric bands for case a (blue lines), case b (orange lines) and case c (purple lines). The darker grey parts correspond to the inner Horsehead where AMM and AMMI grains are used in cases b and c, respectively.

an increase in $M_{\mathrm{a}-\mathrm{C}} / M_{\mathrm{H}}$ increases the extinction significantly. As $M_{\mathrm{a}-\mathrm{C}} / M_{\mathrm{H}}$ increases, the radiation field is therefore increasingly damped at the NIR PP, and fewer photons are available at the FIR PP to heat the larger grains. Panel $\mathrm{j}$ shows that the wavelength associated with the highest emission shifts towards longer wavelengths with an increase in $M_{\mathrm{a}-\mathrm{C}} / M_{\mathrm{H}}$. This means that dust emission in the far-IR varies with $M_{\mathrm{a}-\mathrm{C}} / M_{\mathrm{H}}$, as a result of radiative transfer effects.

The other changes in the spectra are due to variations in dust properties and are explained in Sect. 3.2.

\subsection{With evolved grains}

Previously, we used only CM grains throughout the Horsehead. To study the influence of dust evolution on the emission across the Horsehead, we used CM grains with modified size distributions (i.e. CM grains with values of $M_{\mathrm{a}-\mathrm{C}} / M_{\mathrm{H}}, a_{\mathrm{min}, \mathrm{a}-\mathrm{C}}$, and $\alpha$ that differ from the diffuse ISM) in the outer part of the Horsehead where the dust is likely to be more diffuse ISM-like, and aggregate grains (AMM, AMMI) above a density threshold of $7 \times 10^{4} \mathrm{H} \mathrm{cm}^{-3}$, where dust grains are assumed to be coagulated. In order to simplify our study, we define three different cases depending on the dust we use:

- Case a: CM grains with modified size distributions throughout the Horsehead.

- Case b: CM grains with modified size distributions in the outer part of the Horsehead and AMM in the inner part of the Horsehead.

- Case c: CM grains with modified size distributions in the outer part of the Horsehead and AMMI in the inner part of the Horsehead.

The Dust-modelled emission profiles for the three cases are shown in Fig. 6.

Because the highest emission in the near- and mid-infrared is located in the outer part of the Horsehead, the dust emission at these wavelengths is not modified because we always use modified CM grains. The dust emission in the far-infrared increases when coagulated (AMM and AMMI) dust grains are used because they are more emissive. AMMI grains are more emissive than AMM grains because the dust mass in AMMI grains is higher than in AMM grains because of the ice mantle.

\section{Comparison with observations}

In this section we constrain our dust model with the observations. First, we present our results using diffuse ISM-like dust (i.e. CM grains); second, we introduce the method we use in the following parts; third, we constrain the four parameters $M_{\mathrm{a}-\mathrm{C}} / M_{\mathrm{H}}, a_{\mathrm{min}, \mathrm{a}-\mathrm{C}}, \alpha$, and $l_{\mathrm{PDR}}$ for the three cases of evolved grains as defined in Sect. 4.4 and across the three cuts (see Fig. 1).

\subsection{Diffuse case}

The results are shown in Fig. 7. The ten upper panels correspond to the modelled emission across the Horsehead using CM grains, with $l_{\mathrm{PDR}}$ varying from 0.1 to $0.5 \mathrm{pc}$, for the ten photometric bands. The observed emission is shown for cut 2 . The bottom panels show the corresponding ratios of the highest observed and modelled intensities.

Regardless of the cut considered, it is not possible to simultaneously fit the observations in all the photometric bands (see Fig. 7, upper panel), independently of the $l_{\mathrm{PDR}}$ value. With $l_{\mathrm{PDR}}=0.1 \mathrm{pc}$, we are able to roughly reproduce the observations in the near- and mid-infrared, but in the far-infrared, the modelled dust emission is too low by a factor $\sim 10$ (see Fig. 7, bottom panels). With $l_{\mathrm{PDR}}=0.5 \mathrm{pc}$, we are able to reproduce the observations in the far-infrared but in the near- and midinfrared, the modelled dust emission is too high by a factor of at least $\sim 10$.

If $l_{\mathrm{PDR}}$ is higher than $0.10 \mathrm{pc}$ (see Sect. 2.3), near- and midinfrared modelled dust emission will always be too high, which implies that reducing the dust abundance that is responsible for the emission at these wavelengths decreases the a-C dust-togas ratio, $M_{\mathrm{a}-\mathrm{C}} / M_{\mathrm{H}}$ (see Sect. 4.3). On the other hand, the ratio between the modelled dust emission and the observations is not the same in the five near- and mid-IR bands. The shape of the spectrum therefore needs to be changed by varying $a_{\text {min, a-C }}$ and $\alpha$ (see Sect. 4.3).

To summarise, it is not possible to reproduce the observations across any of the three cuts in the Horsehead for any value of $l_{\mathrm{PDR}}$ using diffuse ISM-like dust alone. We must therefore consider evolved dust. 

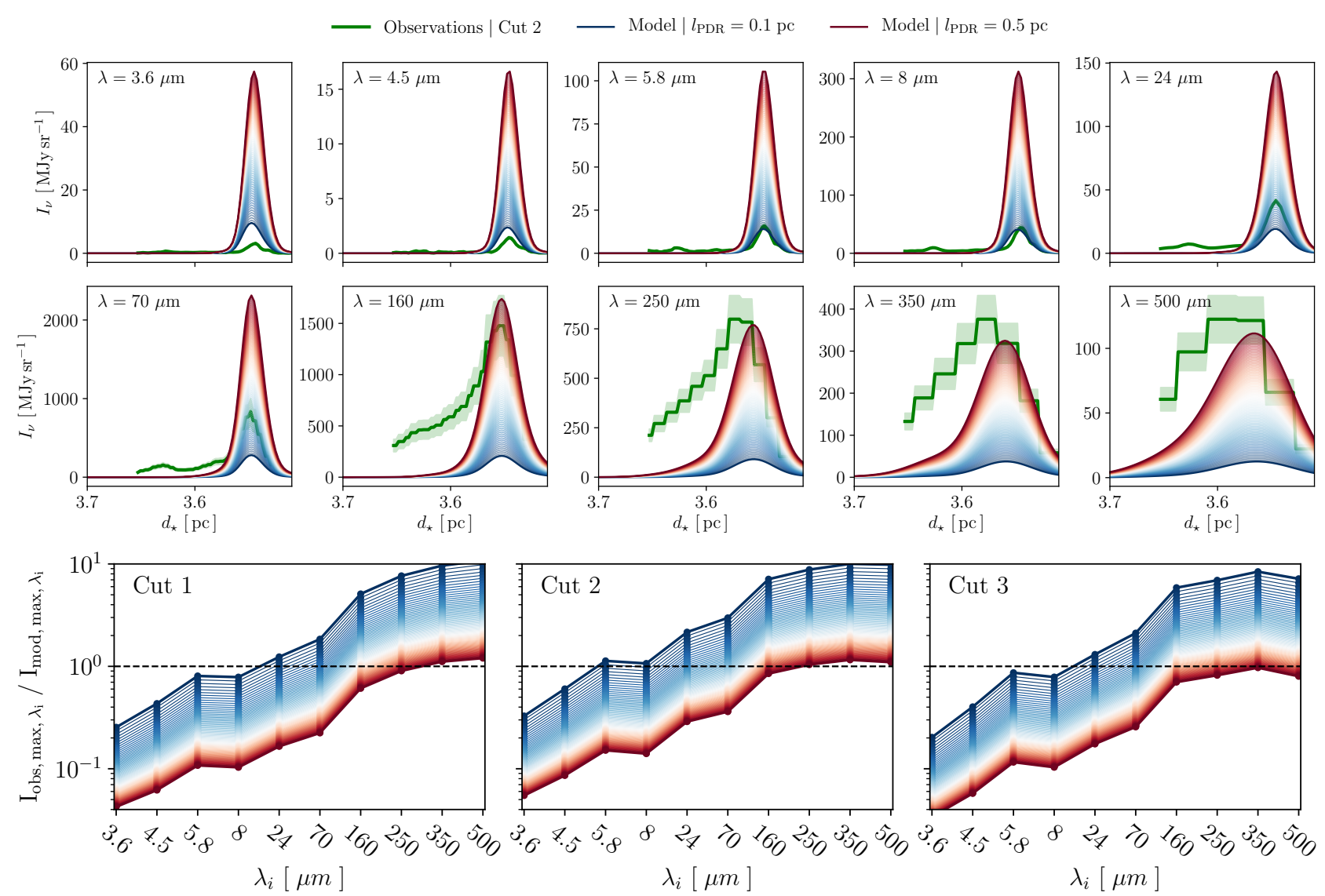

Fig. 7. Top: modelled dust emission for $l_{\mathrm{PDR}}$ varying from $0.1 \mathrm{pc}$ (blue lines) to $0.5 \mathrm{pc}$ (red lines) in steps of $0.01 \mathrm{pc}$ for the ten photometric bands with diffuse ISM-like dust. The observed dust emission for cut 2 is shown by green lines. Bottom: ratio between the highest observed and modelled dust emissions in the ten photometric bands for the three cuts and $l_{\text {PDR }}$ varying from $0.01 \mathrm{pc}$ (blue lines) to $0.5 \mathrm{pc}$ (red lines) in steps of $0.01 \mathrm{pc}$.

\subsection{Method}

To reduce computation time, instead of exploring the $4 \mathrm{D}$ space defined by $M_{\mathrm{a}-\mathrm{C}} / M_{\mathrm{H}}, a_{\mathrm{min}, \mathrm{a}-\mathrm{C}}, \alpha$, and $l_{\mathrm{PDR}}$, we explored the 3D space defined by $M_{\mathrm{a}-\mathrm{C}} / M_{\mathrm{H}}, a_{\min , \mathrm{a}-\mathrm{C}}$, and $\alpha$ as variation in $l_{\text {PDR }}$ does not affect the shape of the dust spectrum (see Sect. 4.2), in contrast to variations in $M_{\mathrm{a}-\mathrm{C}} / M_{\mathrm{H}}, a_{\mathrm{min}, \mathrm{a}-\mathrm{C}}$, and $\alpha$ (see Sect. 4.3). Therefore, $l_{\mathrm{PDR}}$ can be adjusted after the fact.

Adjusting the shape of the modelled dust spectra to the observed dust spectra means that the ratio between $I_{\mathrm{obs} \text {, max }}(i)$ and $I_{\text {mod, max }}(i)$ has to be roughly the same in every band. Therefore we minimised the following parameter:

$\chi^{2}=\sum_{i \in \text { filters }}\left(\frac{X_{i}-\mu}{\sigma_{i}}\right)^{2}$

with

$X_{i}=\frac{I_{\mathrm{obs}, \max }(i)}{I_{\mathrm{mod}, \max }(i)} ; \quad \sigma_{i}=r_{\mathrm{obs}}(i) X_{i} ; \quad \mu=\left\langle X_{i}\right\rangle_{i \in \text { filters }}$

where $r_{\mathrm{obs}}$ is the relative error for each filter and defined in Sect. 2.2 and $I_{\mathrm{obs}, \max }(i)=\max \left(I_{\mathrm{obs}, i}(z)\right)$ with $I_{\mathrm{obs}, i}(z)$, the dust observed in the $i$ th band at the position $z$ along the cut.

The following procedure was thus applied. We constrained $M_{\mathrm{a}-\mathrm{C}} / M_{\mathrm{H}}, a_{\mathrm{min}, \mathrm{a}-\mathrm{C}}$ and $\alpha$ with a fixed $l_{\mathrm{PDR}}$ in order to adjust the shape of the modelled dust spectrum to the observed dust spectrum by minimising $\chi^{2}$. We used the dust properties $\left(M_{\mathrm{a}-\mathrm{C}} / M_{\mathrm{H}}\right.$, $a_{\min , \mathrm{a}-\mathrm{C}}$ and $\alpha$ ) associated with $\chi_{\min }^{2}$ (i.e. the minimum value of $\chi^{2}$ in the 3D space defined by $M_{\mathrm{a}-\mathrm{C}} / M_{\mathrm{H}}, a_{\mathrm{min}, \mathrm{a}-\mathrm{C}}$, and $\alpha$ ), and we adjusted the overall modelled dust spectrum to the observed dust spectrum by multiplying the flux in all bands by the same factor to obtain $\mu=1$, which constrains $l_{\mathrm{PDR}}$.

We chose to remove the $\operatorname{IRAC}_{4.5}$ and $\mathrm{PACS}_{70}$ bands because it is not possible to simultaneously fit the observations in the ten bands with these two included. We discuss this decision further in Sect. 6.1.

In contrast to cut 2 , along which there is only one maximum in the near- and mid-IR, cuts 1 and 3 are composed of two maxima. The far-IR contains only one maximum because the spatial resolution is not sufficient to separate these two maxima. Cuts 1 and 3 contain two maxima and we chose to fit the brightest peak in each cut. We discuss this choice in Sect. 6.2.

\subsection{Constrain $I_{P D R}$ and dust properties $M_{a-C} / M_{H}, a_{\min , a-C}$, and $\alpha$}

First, we studied the $\chi^{2}$ distribution in the 3D space $\left(M_{\mathrm{a}-\mathrm{C}} / M_{\mathrm{H}}\right.$, $a_{\text {min, a-C }}$, and $\alpha$ ) for each of the three cuts and the three cases in order to obtain the best set of parameters in these nine cases. The 3D space is defined as follows:

1. $M_{\mathrm{a}-\mathrm{C}} / M_{\mathrm{H}}$ varies from $0.001 \times 10^{-2}$ to $0.041 \times 10^{-2}$ in steps of $0.002 \times 10^{-2}$.

2. $a_{\mathrm{min}, \mathrm{a}-\mathrm{C}}$ varies from 5 to $10 \mathrm{~nm}$ in steps of $0.25 \mathrm{~nm}$.

3. $\alpha$ varies from -13 to -3 in steps of 0.5 .

Second, we studied the $\chi^{2}$ distribution in 2D space $\left(a_{\mathrm{min}, \mathrm{a}-\mathrm{C}}\right.$ and $\alpha),\left(M_{\mathrm{a}-\mathrm{C}} / M_{\mathrm{H}}\right.$ and $\left.a_{\mathrm{min}, \mathrm{a}-\mathrm{C}}\right)$, and $\left(M_{\mathrm{a}-\mathrm{C}} / M_{\mathrm{H}}\right.$ and $\left.\alpha\right)$. Finally, we concluded with the comparison between the observed and 

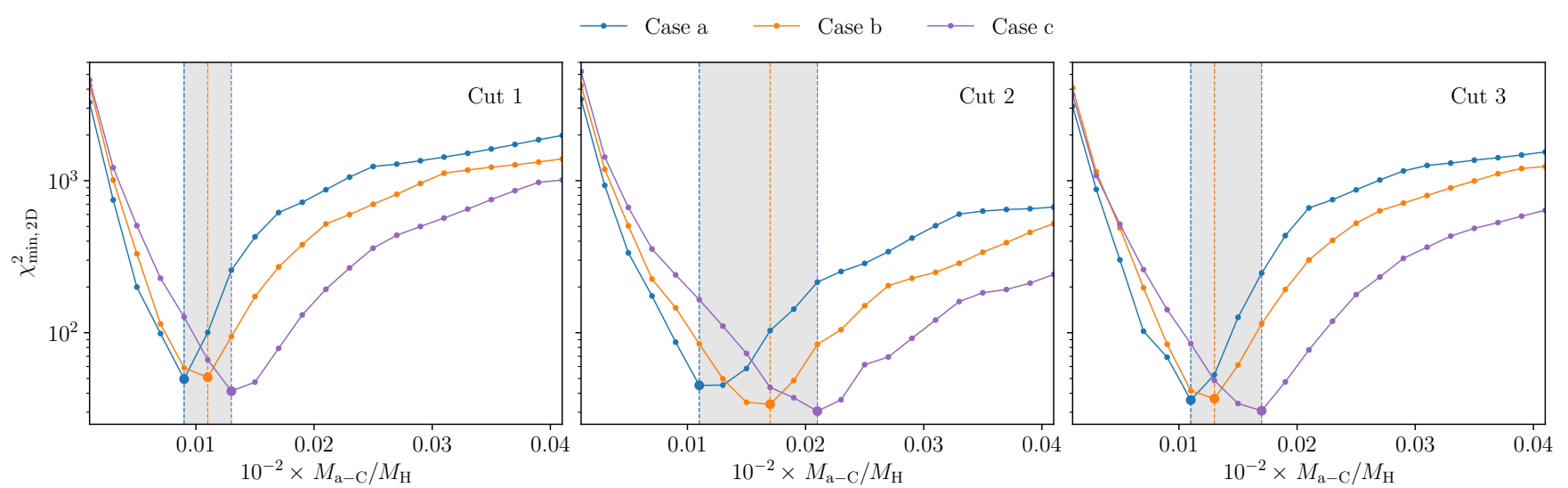

Fig. 8. $\chi_{\min , 2 \mathrm{D}}^{2}\left(M_{\mathrm{a}-\mathrm{C}} / M_{\mathrm{H}}\right)$ as a function of $M_{\mathrm{a}-\mathrm{C}} / M_{\mathrm{H}}$ for cut $1($ left $)$, cut 2 (middle) and cut 3 (right). Blue lines refer to case a, orange lines to case $\mathrm{b}$, and purple lines to case $\mathrm{c}$. Vertical dashed lines correspond to the minima of $\chi_{\min , 2 \mathrm{D}}^{2}$ (i.e. $\chi_{\min }^{2}$ ).

Table 1. Best set of parameters $\left(M_{\mathrm{a}-\mathrm{C}} / M_{\mathrm{H}}, a_{\min , \mathrm{a}-\mathrm{C}}, \alpha\right.$, and $\left.l_{\mathrm{PDR}}\right)$ and the $\chi_{\min }^{2}$ associated with all cuts and cases.

\begin{tabular}{|c|c|c|c|c|c|c|c|c|c|}
\hline & \multicolumn{3}{|c|}{ Case a } & \multicolumn{3}{|c|}{ Case b } & \multicolumn{3}{|c|}{ Case c } \\
\hline & cut 1 & cut 2 & cut 3 & cut 1 & cut 2 & cut 3 & cut 1 & cut 2 & cut 3 \\
\hline $10^{2} \times M_{\mathrm{a}-\mathrm{C}} / M_{\mathrm{H}}$ & 0.009 & 0.011 & 0.011 & 0.011 & 0.017 & 0.013 & 0.013 & 0.021 & 0.017 \\
\hline$a_{\min , \mathrm{a}-\mathrm{C}}[\mathrm{nm}]$ & 0.825 & 0.825 & 0.925 & 0.825 & 0.8 & 0.925 & 0.825 & 0.8 & 0.9 \\
\hline$\alpha$ & -7.0 & -6.0 & -7.5 & -6.5 & -5.5 & -7.5 & -6.5 & -5.5 & -6.5 \\
\hline$l_{\mathrm{PDR}}[\mathrm{pc}]$ & 0.283 & 0.297 & 0.273 & 0.290 & 0.267 & 0.282 & 0.275 & 0.254 & 0.265 \\
\hline$\chi_{\min }^{2}$ & 49.6 & 45.1 & 36.0 & 51.0 & 33.9 & 36.9 & 41.3 & 30.5 & 30.7 \\
\hline
\end{tabular}

modelled dust emission profiles for each of the three cuts and the three cases with the best sets of parameters.

For more clarity, we defined $\chi_{\min , 2 \mathrm{D}}^{2}\left(M_{\mathrm{a}-\mathrm{C}} / M_{\mathrm{H}}\right)$, which is the minimum value of $\chi^{2}$ in $2 \mathrm{D}$ space $\left(a_{\min , \mathrm{a}-\mathrm{C}}\right.$ and $\left.\alpha\right)$ for a given value of $M_{\mathrm{a}-\mathrm{C}} / M_{\mathrm{H}}$. We also defined $\chi_{\min }^{2}$, which is the minimum value of $\chi^{2}$ in 3D space $\left(M_{\mathrm{a}-\mathrm{C}} / M_{\mathrm{H}}, a_{\mathrm{min}, \mathrm{a}-\mathrm{C}}\right.$, and $\left.\alpha\right)$, that is, the lowest value of $\chi_{\min , 2 \mathrm{D}}^{2}\left(M_{\mathrm{a}-\mathrm{C}} / M_{\mathrm{H}}\right)$.

\subsection{1. $\chi^{2}$ distribution in $3 \mathrm{D}$ space $\left(M_{\mathrm{a}-\mathrm{C}} / M_{\mathrm{H}}, \mathrm{a}_{\mathrm{min}, \mathrm{a}-\mathrm{C}}\right.$, and $\left.\alpha\right)$}

Figure 8 shows $\chi_{\min , 2 \mathrm{D}}^{2}\left(M_{\mathrm{a}-\mathrm{C}} / M_{\mathrm{H}}\right)$ and Table 1 summarises these results.

First and foremost, $M_{\mathrm{a}-\mathrm{C}} / M_{\mathrm{H}}$ is between $0.01 \times 10^{-2}$ and $0.03 \times 10^{-2}$, that is, between 6 and 10 times lower than in the diffuse ISM $\left(0.17 \times 10^{-2}\right)$ regardless of the cut or the case considered. Second, $a_{\mathrm{min}, \mathrm{a}-\mathrm{C}}$ is between 0.8 and $0.925 \mathrm{~nm}$, that is between 2 and 2.25 times larger than in the diffuse ISM $(0.4 \mathrm{~nm})$. Third, $\alpha$ is between -7 and -5.5 , that is, between 1.1 and 1.4 times lower than in the diffuse ISM $(-5)$.

Regardless of the cut, $M_{\mathrm{a}-\mathrm{C}} / M_{\mathrm{H}}$ increases from case a to case c (see Fig. 8). In case a, we only used modified CM grains (i.e. $\mathrm{CM}$ grains with values of $M_{\mathrm{a}-\mathrm{C}} / M_{\mathrm{H}}, a_{\min , \mathrm{a}-\mathrm{C}}$, and $\alpha$ that differ from the diffuse ISM) in the outer part and in the inner part of the Horsehead, but we use modified CM grains in the outer part of the Horsehead and AMMI grains in the inner part in case $c$. Because AMMI grains are more emissive in the farIR than CM grains, emission in this wavelength range must decrease to fit the observations, which can be achieved by reducing $l_{\mathrm{PDR}}$ (see Sect. 4.2), hence $l_{\mathrm{PDR}}$ decreases from case a to case $c$. This decrease in $l_{\mathrm{PDR}}$ implies a decrease in emission in the near- and mid-IR, which means that $M_{\mathrm{a}-\mathrm{C}} / M_{\mathrm{H}}$ must increase to counterbalance this variation.

\subsection{2. $\chi^{2}$ distribution in $2 \mathrm{D}$ space $\left(\mathrm{a}_{\min , \mathrm{a}-\mathrm{C}}\right.$ and $\left.\alpha\right)$, $\left(M_{\mathrm{a}-\mathrm{C}} / M_{\mathrm{H}}\right.$ and $\left.\mathrm{a}_{\mathrm{min}, \mathrm{a}-\mathrm{C}}\right)$, and $\left(M_{\mathrm{a}-\mathrm{C}} / M_{\mathrm{H}}\right.$ and $\left.\alpha\right)$}

We show in Fig. 9 the $\chi^{2}$ distribution for cut 2 in 2D space $\left(a_{\mathrm{min}, \mathrm{a}-\mathrm{C}}\right.$ and $\left.\alpha\right),\left(M_{\mathrm{a}-\mathrm{C}} / M_{\mathrm{H}}\right.$ and $\left.a_{\mathrm{min}, \mathrm{a}-\mathrm{C}}\right)$, and $\left(M_{\mathrm{a}-\mathrm{C}} / M_{\mathrm{H}}\right.$ and $\alpha)$. We chose to focus on only one cut because we are interested in the behaviour of the $\chi^{2}$ distribution here, which is the same regardless of the cut.

The most important result is that regardless of the case, there is a unique minimum in all 2D spaces. Moreover, as explained in Sect. 3.2, a decrease in $M_{\mathrm{a}-\mathrm{C}} / M_{\mathrm{H}}$, to first order, is similar to an increase in $a_{\text {min, a-C }}$ and $\alpha$, regarding dust emission in the nearand mid- IR. An increase in $a_{\text {min, a-C }}$ is therefore counterbalanced by a decrease in $\alpha$ to keep low values of $\chi^{2}$, and an increase in $M_{\mathrm{a}-\mathrm{C}} / M_{\mathrm{H}}$ is counterbalanced by an increase in $a_{\mathrm{min}, \mathrm{a}-\mathrm{C}}$ and in $\alpha$, which explains the banana-shape of the low $\chi^{2}$ values in each of the 2D spaces.

Furthermore, from case a to case $\mathrm{c}$, the position of $\chi^{2}$ minimum value moves. From case a to case c, dust emission in the far-IR increases (see Fig. 6), which shows that this effect is counterbalanced by a decrease in $l_{\mathrm{PDR}}$ that also reduces dust emission in the near- and mid-IR. To compensate for this decrease in dust emission in the near- and mid-IR, the value of $M_{\mathrm{a}-\mathrm{C}} / M_{\mathrm{H}}$ associated with the $\chi^{2}$ minimum value increases from case a to case $\mathrm{c}$ in 2D spaces $\left(M_{\mathrm{a}-\mathrm{C}} / M_{\mathrm{H}}\right.$ and $\left.a_{\mathrm{min}, \mathrm{a}-\mathrm{C}}\right)$ and $\left(M_{\mathrm{a}-\mathrm{C}} / M_{\mathrm{H}}\right.$ and $\left.\alpha\right)$. In 2D space $\left(a_{\mathrm{min}, \mathrm{a}-\mathrm{C}}\right.$ and $\left.\alpha\right)$, this effect is counterbalanced by a decrease in $\alpha$ and an increase in $a_{\mathrm{min}, \mathrm{a}-\mathrm{C}}$.

\subsubsection{Comparison between dust-modelled emission and dust-observed emission profiles}

Here, we use the best set of parameters $\left(M_{\mathrm{a}-\mathrm{C}} / M_{\mathrm{H}}, a_{\text {min, a-C }}, \alpha\right.$ and $\left.l_{\mathrm{PDR}}\right)$ that are listed in Table 1 and compare the modelled 
$\chi^{2}$ for Cut $2\left|\mathrm{M}_{\mathrm{a}-\mathrm{C}} / \mathrm{M}_{\mathrm{H}}=0.021 \times 10^{-2}\right|$ Left : Case a $\mid$ Middle : Case b $\mid$ Right : Case c $10^{2}$

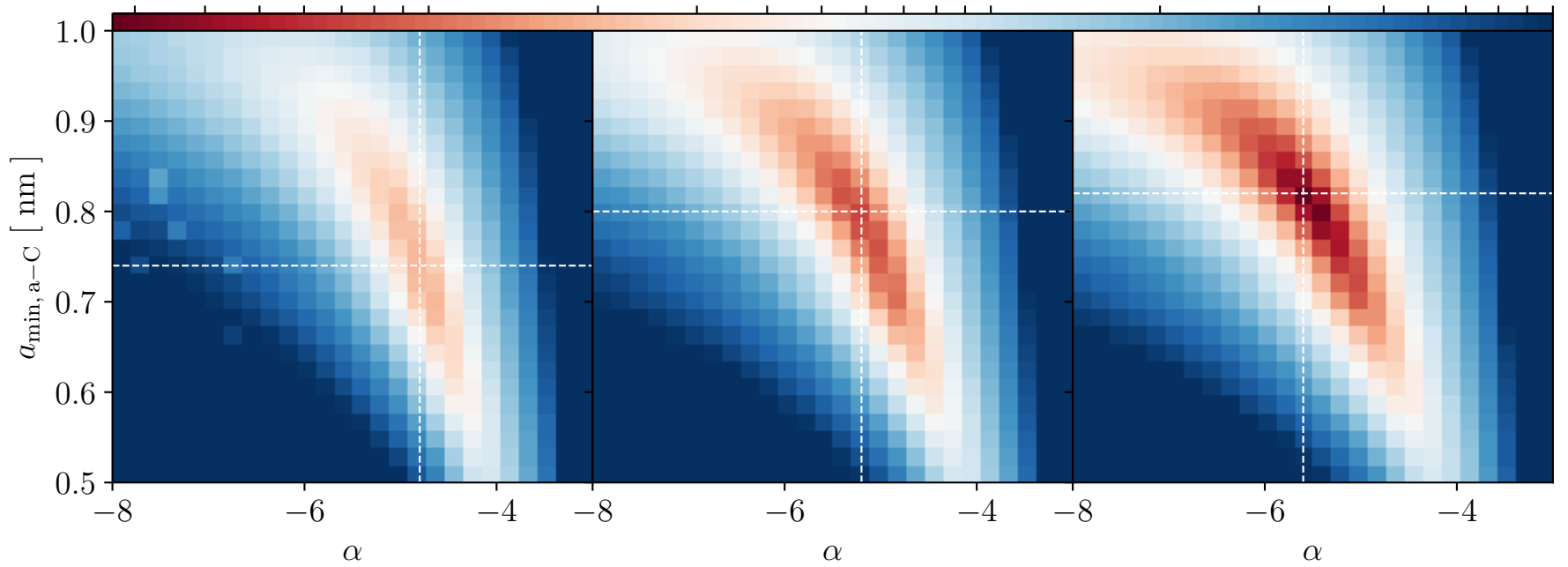

$\chi^{2}$ for Cut $2|\alpha=-5.5|$ Left : Case a $\mid$ Middle : Case b $\mid$ Right : Case c $10^{2}$

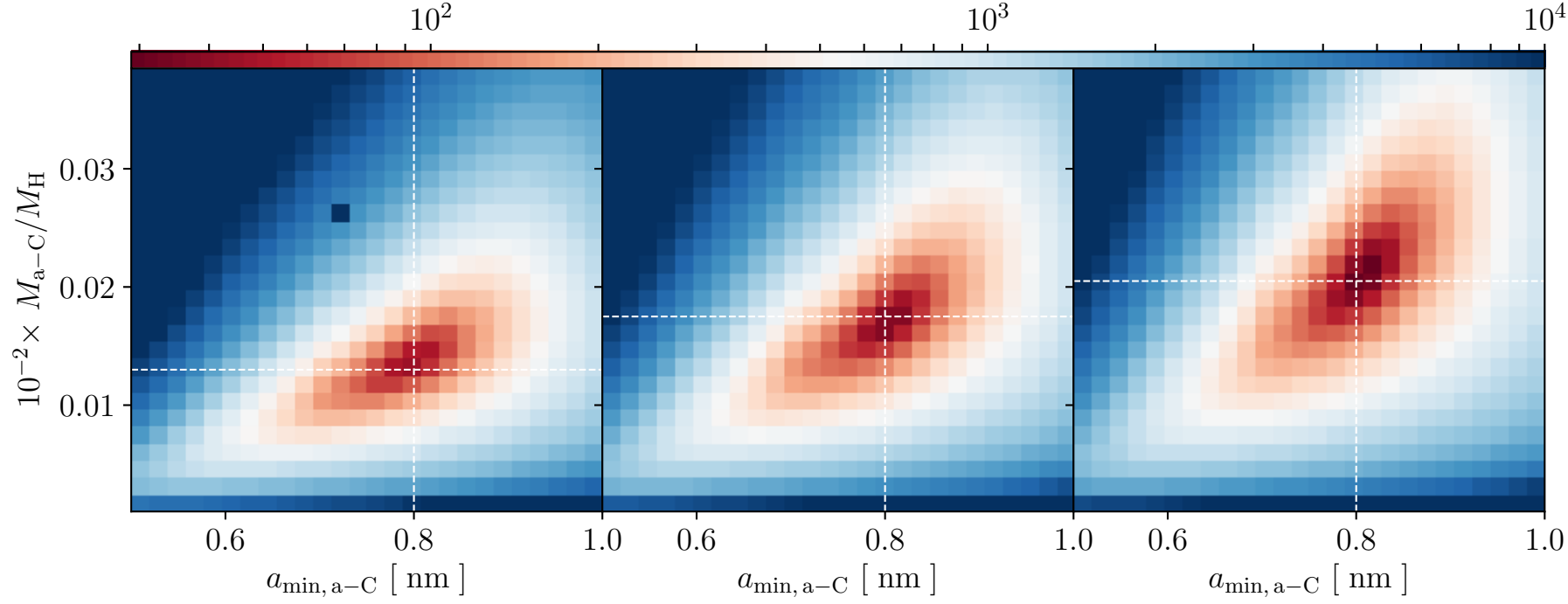

$\chi^{2}$ for Cut $2\left|a_{\min , \mathrm{a}-\mathrm{C}}=0.80 \mathrm{~nm}\right|$ Left $:$ Case a $\mid$ Middle : Case b $\mid$ Right : Case c

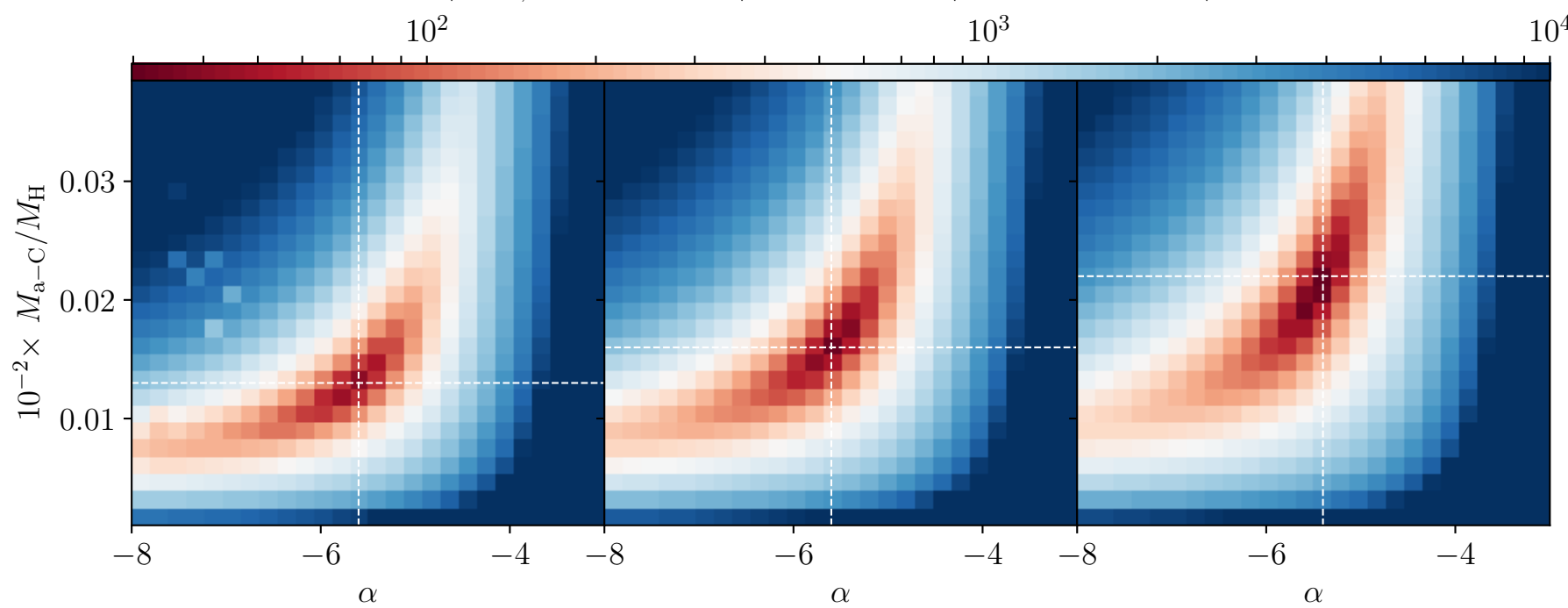

Fig. 9. Top: $\chi^{2}$ in 2D space $\left(a_{\mathrm{min}, \mathrm{a}-\mathrm{C}}\right.$ and $\alpha$ ) for cut 2 with $M_{\mathrm{a}-\mathrm{C}} / M_{\mathrm{H}}=0.021 \times 10^{-2}$. From left to right: subplots correspond to case a (left), case b (middle), and case c (right). Middle: $\chi^{2}$ in $2 \mathrm{D}$ space $\left(M_{\mathrm{a}-\mathrm{C}} / M_{\mathrm{H}}\right.$ and $\left.a_{\mathrm{min}, \mathrm{a}-\mathrm{C}}\right)$ for cut 2 with $\alpha=-5.5$. Bottom: $\chi^{2}$ in $2 \mathrm{D}$ space $\left(M_{\mathrm{a}-\mathrm{C}} / M_{\mathrm{H}}\right.$ and $\left.\alpha\right)$ for cut 2 with $a_{\min , \mathrm{a}-\mathrm{C}}=0.80 \mathrm{~nm}$. 
emission profiles in the ten photometric bands for the three cases with the observed emission profiles in the three cuts (see Fig. 10). We focus on three aspects: the highest intensity in each of the ten bands, the position of these maxima, and the width of these profiles.

In the near- and mid-IR, except in $\mathrm{IRAC}_{4.5}$, the maximum emission is well reproduced, regardless the case or the cut. In $\mathrm{PACS}_{70}$, although the maximum of emission is never reproduced, the discrepancy between the maximum modelled emission and the maximum observed emission decreases from case a to case c. From $\mathrm{SPIRE}_{250}$ to $\mathrm{SPIRE}_{500}$, the maximum emission is in the error bars, regardless of the case or the cut, and the discrepancy between the maximum modelled emission and the maximum observed emission decreases from case a to case c. In $\mathrm{PACS}_{160}$, the maximum emission is within the error bars only for case $\mathrm{c}$ for cuts 2 and 3 , but never for cut 1 , regardless of the case.

The position of the maximum emission is well reproduced from $\mathrm{IRAC}_{3.6}$ to $\mathrm{PACS}_{70}$ regardless of the cut and the case. Cut 1 shows a small discrepancy between the position of the maximum emission and the position of the observed emission from PACS $_{70}$ to SPIRE 500 . For cut 2, there is the same discrepancy in SPIRE $_{350}$ and SPIRE $_{500}$, regardless of the case. For cut 3 , all the positions are well reproduced.

The width of the profiles are well reproduced from IRAC $_{3.6}$ to PACS $_{160}$, but slightly different from SPIRE 250 to SPIRE $_{500}$. This might be due to large structures in the Horsehead.

To summarise, the observed dust emission is well reproduced in the near- and mid-IR, except in IRAC $_{4.5}$, regardless of the case and the cut. In the far-IR, the discrepancy between observed dust emission and modelled dust emission decreases from case a to case c.

\section{Discussion}

First, we discuss the discrepancy between the dust-modelled emission and the dust-observed emission in IRAC $_{4.5}$ and in PACS $_{70}$; second, the results obtained are described; third, we propose a scenario of dust evolution in agreement with the results obtained. We conclude with a discussion of dustprocessing timescales in support of this scenario.

\subsection{Discrepancy in IRAC 4.5 and $P A C S_{70}$}

In IRAC $_{4.5}$, the modelled dust emission is always overestimated (see Fig. 10) by a factor 2-4. Because this filter covers the dust continuum and the wings of the IR bands from a-C:H nanograin, this suggests that the wings of the IR bands in this region are different (i.e. weaker and/or narrower, see e.g. Boutéraon et al. 2019 for more details about the variability of the IR-band widths) from those in the diffuse ISM. We here study dust that evolves from dense cloud dust in response to interaction with UV photons.

Moreover, a-C:H nano-grain freshly produced may not yet have had time to be entirely photo-processed and therefore have a high band-to-continuum ratio because of their high fraction of aliphatic bonds, as opposed to aromatic bonds. As discussed in Jones et al. (2013), this requires a-C:H nano-grain with a band gap larger than $0.1 \mathrm{eV}$, the value adopted in the diffuse ISM.

However, because we lack dust spectroscopic information of the Horsehead in the near-IR, we are unable to answer to these previous questions. In the near future, JWST spectroscopic data should allow us to understand such changes in the structure of a-C:H nano-grains.

In $\mathrm{PACS}_{70}$, models always overestimate the emission (see Fig. 10) by a factor 3-4. This suggests that large grains (a-Sil/a$\mathrm{C}$ and $\mathrm{a}-\mathrm{C}: \mathrm{H} / \mathrm{a}-\mathrm{C}$ for case $\mathrm{a}, \mathrm{AMM}$ grains for case $\mathrm{b}$ and AMMI grains for case c) are somewhat too warm and not emissive enough. This is supported by recent laboratory experiments in which the mass absorption coefficient of silicates in the far-IR is larger (up to an order of magnitude) than the coefficient that are currently used in THEMIS (see Fig. 5 in Demyk et al. 2017). As a consequence, the large grains we used here are probably not emissive enough. The incorporation of these new laboratory results in THEMIS will most likely reduce the discrepancy in PACS $_{70}$.

\subsection{Main results}

Using the 3D radiative transfer code SOC together with the dust model THEMIS, we can reproduce the Horsehead observations in eight of the ten photometric bands of Spitzer and Herschel.

The main results for the outer part of the Horsehead are the following:

1. The nano-grain (i.e. a-C grains) dust-to-gas mass ratio, $M_{\mathrm{a}-\mathrm{C}} / M_{\mathrm{H}}$, is 6-10 times lower than in the diffuse ISM.

2. The minimum size of the nano-grain, $a_{\mathrm{min}, \mathrm{a}-\mathrm{C}}$, is 2 to 2.25 times larger than in the diffuse ISM.

3. The power-law exponent of the nano-grain size distribution, $\alpha$, is 1.1-1.4 times lower than in the diffuse ISM, that is, the size distribution is steeper.

The best size distributions for the three cuts and case $c$ are shown in Fig. 11. For the inner part of the Horsehead, we tested three different types of dust, diffuse ISM-like dust (CM) with modified size distributions in case a, aggregates of grains (AMM) in case $b$, and aggregates of grains with ice mantles (AMMI) in case c. At long wavelengths (from PACS ${ }_{160}$ to SPIRE $_{500}$ ), the results are significantly better when we used AMMI instead of $\mathrm{CM}$ grains. For $\mathrm{PACS}_{70}$, even though we are unable to reproduce the observed emission with our model, using aggregates (AMM or AMMI) instead of diffuse ISM-like dust (CM) with modified size distributions, significantly improved the fit in this band.

The length of the Horsehead along the line of sight, $l_{\mathrm{PDR}}$, is found to be within the range of 0.26 and $0.30 \mathrm{pc}$. This agrees with previous gas studies (Habart et al. 2005).

We note that $M_{\mathrm{a}-\mathrm{C}} / M_{\mathrm{H}}$ is higher for cut 2 than cuts 1 and 3 (see Table 1). In these two cuts, the peak in the FIR is composed of two substructures that are spatially unresolved, in constrast to the near- and mid-IR. Consequently, $l_{\mathrm{PDR}}$ has to be larger for cuts 1 and 3 , in contrast to what would have been expected if these two peaks had been resolved. Because an increase in $l_{\mathrm{PDR}}$ implies an increase in the near- and mid-infrared emission, it is therefore required to decrease $M_{\mathrm{a}-\mathrm{C}} / M_{\mathrm{H}}$. We conclude that the different values of $M_{\mathrm{a}-\mathrm{C}} / M_{\mathrm{H}}$ in Table 1 for the three cuts do not reveal any difference in dust properties.

\subsection{Dust evolution scenario}

Our results show significant variations of the dust size distribution, and in the following, we outline a possible scenario of dust evolution across the Horsehead interface. Because of the strong incident radiation field, we assume that the dominant process is the exposure of dust grains from the dense molecular cloud (the inner region) to the UV light of $\sigma$-Ori. This suggests two main photo-processing sequences: (i) the partial fragmentation 
- Observations $\mid$ Cut $1 \_$Model $\mid$Case a $\longrightarrow$ Model $\mid$ Case b $\quad$ Model | Case c
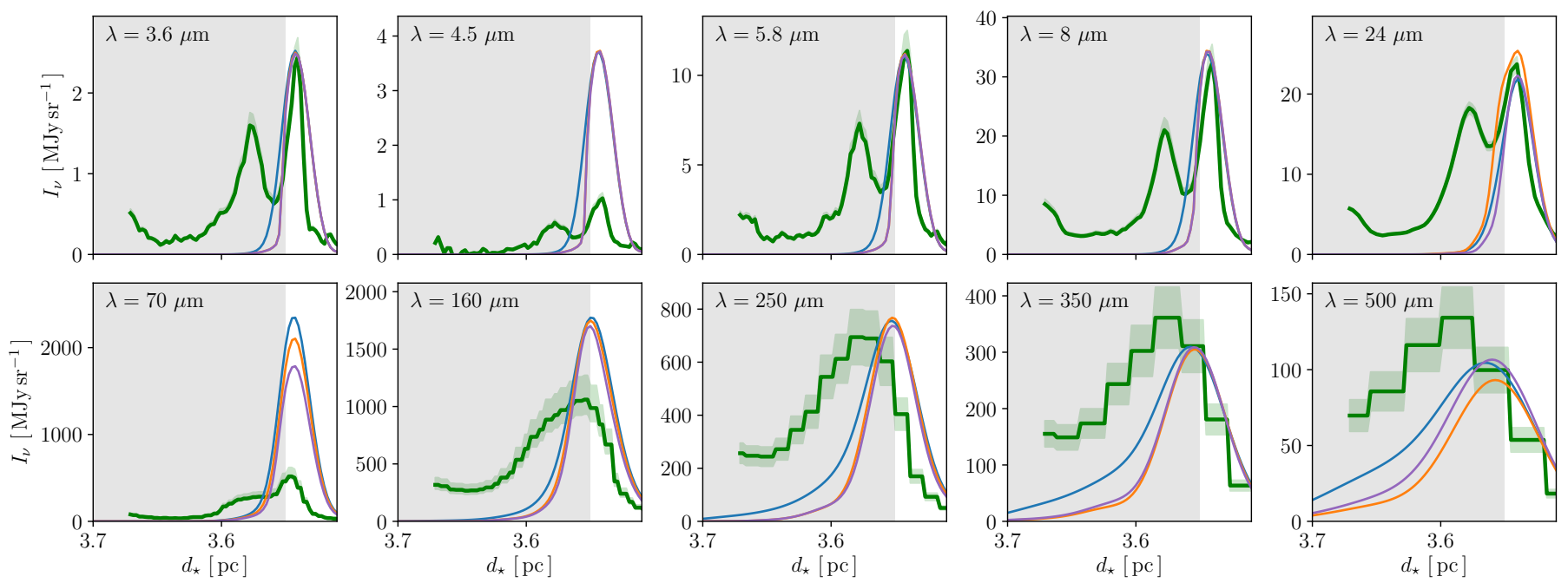

- Observations | Cut $2-$ Model | Case a $\longrightarrow$ Model | Case b $\longrightarrow$ Model | Case c
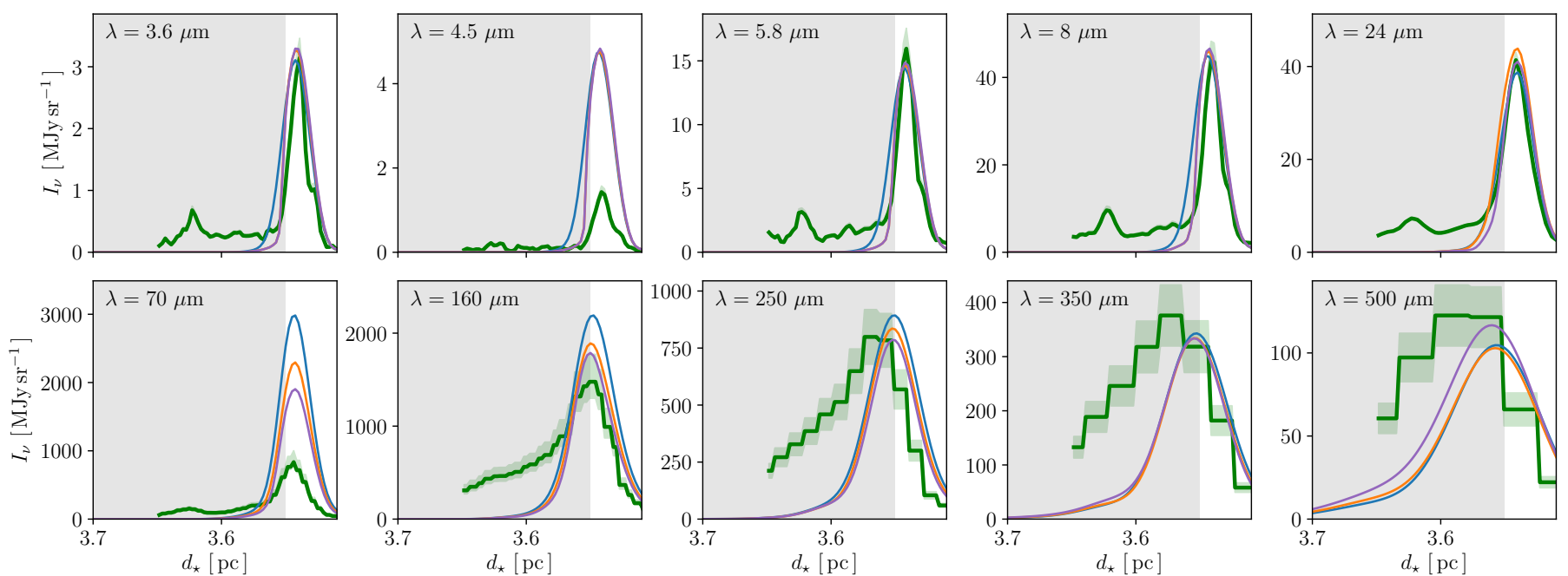

- Observations $\mid$ Cut $3 \simeq$ Model $\mid$ Case a $—$ Model $\mid$ Case $b \quad$ Model $\mid$ Case c
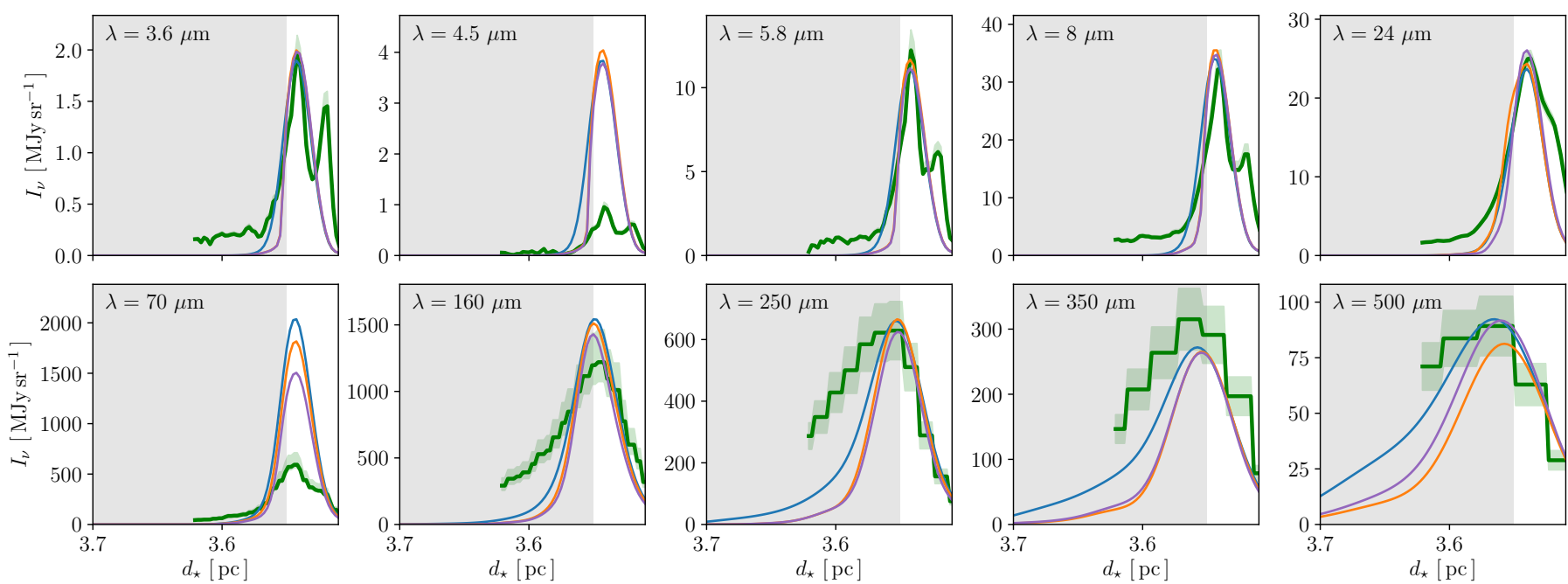

Fig. 10. Top: comparison between observed emission profiles for cut 1 (green line) with modelled emission profiles obtained with the best set of parameters (see Table 1) for case a (blue line), case b (orange line), and case c (purple line). Middle: same for cut 2. Bottom: same for cut 3. The grey parts correspond to the inner Horsehead, where AMM and AMMI grains are used in case b and case c, respectively. 


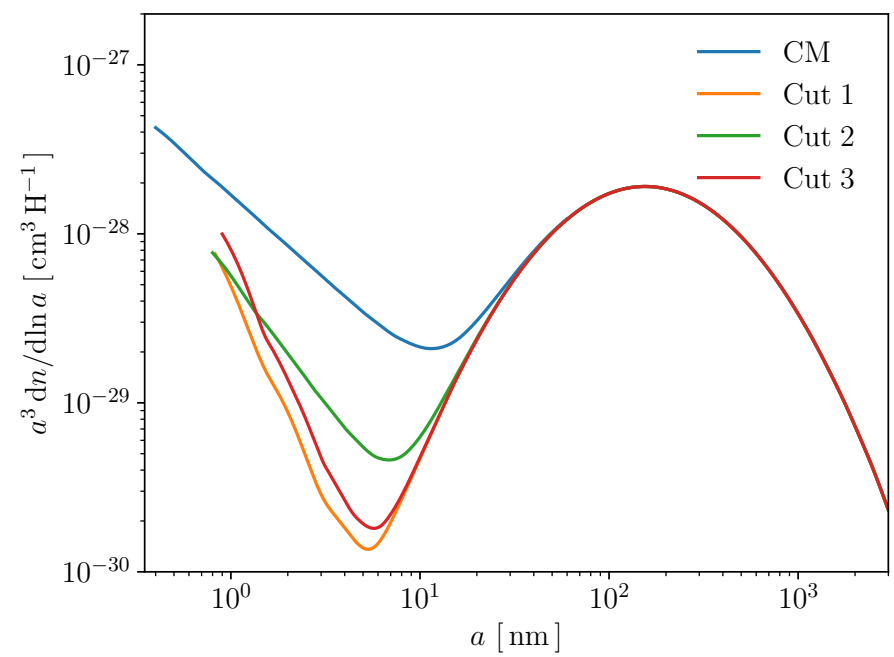

Fig. 11. Size distribution for diffuse ISM-like dust (CM) in blue. Modified size distributions using the best set of parameters (see Table 1) in case $\mathrm{c}$ for cut 1 (orange), cut 2 (green), and cut 3 (red).

of aggregate grains from the inner region and (ii) the destruction of the smallest a-C:H nano-grain. We discuss the significance of these sequences by comparing their timescales to the advection timescale $\tau_{\mathrm{a}}$, that is, the time that the incident UV light needs to heat up and dissociate the molecular gas at the cloud border.

The advection timescale is defined as $\tau_{\mathrm{a}}=L / v_{\mathrm{DF}}$ where $L \sim$ $0.05 \mathrm{pc}$ is the width of the outer part of the Horsehead, and $v_{\mathrm{DF}} \sim$ $0.5 \mathrm{~km} \mathrm{~s}^{-1}$ is the velocity of the dissociation front (Hollenbach $\&$ Tielens 1999). With these values, we find $\tau_{\mathrm{a}} \sim 10^{5} \mathrm{yr}$.

Due to the lack of studies, we took the photo-darkening timescale as a lower limit to photo-fragmentation of aggregate grains and photo-destruction of a-C nano-grain, described by $\tau_{\mathrm{ph}}$. Photo-darkening involves the dissociation of $\mathrm{CH}$-bonds, a process that is more likely faster than the breaking of CC-bonds that must occur in photo-fragmentation (Jones \& Habart 2015). We therefore express $\tau_{\mathrm{ph}}$ at the cloud edge in terms of the photo-darkening rate $\Lambda_{\text {pd }}$ (Jones et al. 2014),

$\tau_{\mathrm{ph}} \simeq \Lambda_{\mathrm{pd}}^{-1}=\frac{1}{\sigma_{\mathrm{CH}} F_{\mathrm{UV}}^{0} Q_{\mathrm{abs}}(a) \epsilon(a)}$,

where $F_{\mathrm{UV}}^{0} \simeq 3.8 \times 10^{9}$ photons s $\mathrm{cm}^{-1}$ is the unattenuated $\mathrm{UV}$ field, $\epsilon(a)=\min \left(1, \frac{2}{a[\mathrm{~nm}]}\right)$ is the size-dependent photo-darkening efficiency, $\sigma_{\mathrm{CH}} \simeq 10^{-19} \mathrm{~cm}^{2}$ is the $\mathrm{CH}$ bond photo-dissociation cross-section, and $Q_{\text {abs }}(a)$ is the dust absorption efficiency which depends almost solely on the radius in the UV range. In the case of AMMI, $\tau_{\mathrm{ph}}$ is larger in reality because the ice mantle needs to be vaporised first, but we did not take this effect into account because the time $\tau_{\text {ph }}$ we estimate is already a lower limit.

We show $\tau_{\mathrm{ph}}(a)$ in Fig. 12 for CM, AMM, and AMMI grains. As discussed in Ysard et al. (2016), more than 50\% of the AMM(I) dust mass is contained in grains larger than $250 \mathrm{~nm}$. This figure shows that aggregate grains can be photo-fragmented because $\tau_{\mathrm{ph}} \sim \tau_{\mathrm{a}}$. Moreover, a-C nano-grain can be efficiently destroyed as $\tau_{\mathrm{ph}}<\tau_{\mathrm{a}}$ for a-C nano-grain. Similar results were found by Alata et al. (2014), from laboratory experiments on a$\mathrm{C}: \mathrm{H}$ grain analogues that were later applied to the Horsehead (Alata et al. 2015).

From this analysis emerges the following scenario. Within an advection timescale, the a-C nano-grain formed by fragmentation of aggregate grains are also partially destroyed by UV

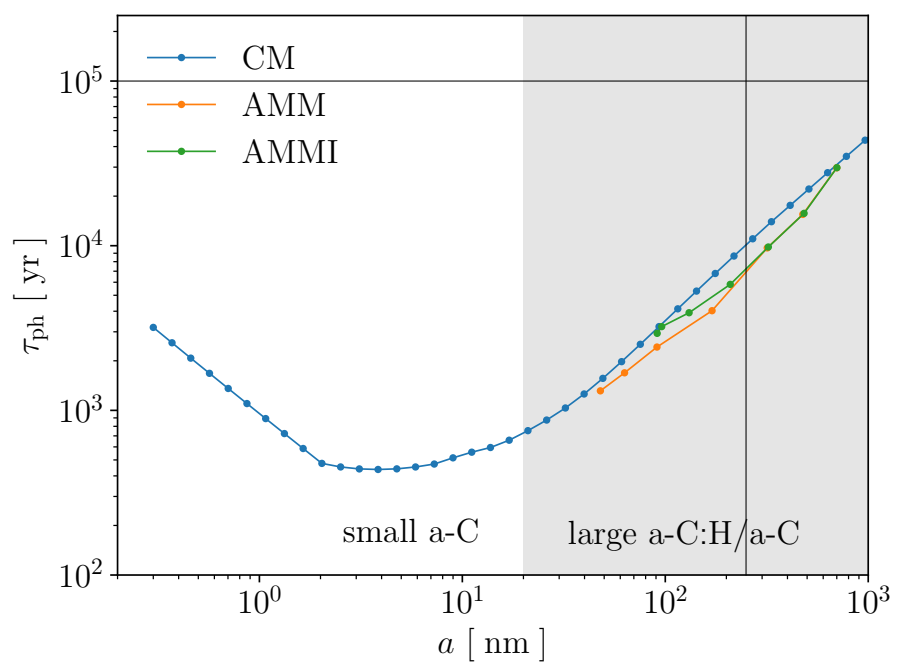

Fig. 12. Photo-fragmentation timescale at the Horsehead edge as a function of the grain radius, $a$. The blue line refers to $\mathrm{CM}$ grains, the orange line to AMM grains, and the green line to AMMI grains. The horizontal black line corresponds to the advection timescale $\tau_{\mathrm{a}} \sim 10^{5} \mathrm{yr}$ in the outer part of the Horsehead. The vertical black line corresponds to the limit at $250 \mathrm{~nm}$ beyond which more than $50 \%$ of the AMM(I) dust mass is contained (Ysard et al. 2016). The darker grey part corresponds to sizes that cover large a-C:H/a-C grains.

photons. This naturally explains the depletion of a-C:H grains around $a=10 \mathrm{~nm}$ seen in Fig. 11. We note that the size distribution of these freshly formed small grains is significanlty different from the diffuse ISM case (blue curve in Fig. 11). This evolved size distribution could reflect the photo-evaporated layer described by Bron et al. (2018).

\section{Conclusion}

With Herschel and Spitzer data, we studied the Horsehead using ten photometric bands from 3.6 to $500 \mu \mathrm{m}$, that cover the entire dust spectrum. We modelled the dust emission across the Horsehead using the THEMIS dust model together with the 3D radiative transfer code $S O C$.

We show that it is not possible to reproduce the observations in the Horsehead using dust grains from the diffuse ISM. It is therefore required to modify their size distributions and compositions. Dust therefore evolves across the Horsehead.

In the outer part of the Horsehead, the a-C nano-grain dustto-gas ratio is 6-10 times lower and their minimum size is 2-2.25 times larger than in the diffuse ISM. The power law of the size distribution is steeper than in the diffuse ISM. In the inner part of the Horsehead, we showed that using aggregate grains with or without ice mantles significantly reduces the discrepancy between our model and the observations. The discrepancy between the observations and our model at $4.5 \mu \mathrm{m}$ might be due to the shape of the aromatic band wings, whence the overestimation of the dust-modelled emission. We also find that large grains are too warm because our modelled dust emission at $70 \mu \mathrm{m}$ is overestimated. However, laboratory studies show that large silicate grains are more emissive than those used in dust models and are therefore cooler. These new results will soon be implemented in THEMIS.

Based on a timescale analysis, we propose a scenario where the a-C nano-grain form by the partial photo-fragmentation of aggregate grains and are processed by the UV photons, leading to a size distribution depleted in grains of size from 5 to $10 \mathrm{~nm}$. 
In the denser regions of the Horsehead, the dust composition is typical of dense clouds.

Spectroscopic observations of the Horsehead are required to proceed on the structure and size distribution of a-C nanograin. Observations with the JWST will for the first time spatially resolve the individual IR dust signatures across the Horsehead, offering an unprecedented look at the evolution of the interstellar matter in photon-dominated regions.

Acknowledgements. We thank the anonymous referee for very helpful suggestions and comments. We also would like to thank the CNES and the P2IO LabeX for supporting Thiebaut Schirmer PhD work. This work was supported by the Programme National "Physique et Chimie du Milieu Interstellaire" (PCMI) of CNRS/INSU with INC/INP co-funded by CEA and CNES. HIPE is a joint development by the Herschel Science Ground Segment Consortium, consisting of ESA, the NASA Herschel Science Center, and the HIFI, PACS and SPIRE consortia. PACS has been developed by a consortium of institutes led by MPE (Germany) and including UVIE (Austria); KU Leuven, CSL, IMEC (Belgium); CEA, LAM (France); MPIA (Germany); INAF-IFSI/OAA/OAP/OAT, LENS, SISSA (Italy); IAC (Spain). This development has been supported by the funding agencies BMVIT (Austria), ESA-PRODEX (Belgium), CEA/CNES (France), DLR (Germany), ASI/INAF (Italy), and CICYT/MCYT (Spain). SPIRE has been developed by a consortium of institutes led by Cardiff Univ. (UK) and including Univ. Lethbridge (Canada); NAOC (China); CEA, LAM (France) IFSI, Univ. Padua (Italy); IAC (Spain); Stockholm Observatory (Sweden); Imperial College London, RAL, UCL-MSSL, UKATC, Univ. Sussex (UK); Caltech, JPL, NHSC, Univ. Colorado (USA). This development has been supported by national funding agencies: CSA (Canada); NAOC (China); CEA, CNES, CNRS (France); ASI (Italy); MCINN (Spain); SNSB (Sweden); STFC, UKSA (UK); and NASA (USA).

\section{References}

Abergel, A., Boulanger, F., Mizuno, A., \& Fukui, Y. 1994, ApJ, 423, L59 Abergel, A., Teyssier, D., Bernard, J. P., et al. 2003, A\&A, 410, 577 Abergel, A., Arab, H., Compiègne, M., et al. 2010, A\&A, 518, L96 Alata, I., Cruz-Diaz, G. A., Muñoz Caro, G. M., \& Dartois, E. 2014, A\&A, 569, A119

Alata, I., Jallat, A., Gavilan, L., et al. 2015, A\&A, 584, A123

Anthony-Twarog, B. J. 1982, AJ, 87, 1213

Arab, H. 2012, PhD thesis, Université Paris-Sud, Orsay

Arab, H., Abergel, A., Habart, E., et al. 2012, A\&A, 541, A19

Bakes, E. L. O., \& Tielens, A. G. G. M. 1994, ApJ, 427, 822

Bernard, J. P., Abergel, A., Ristorcelli, I., et al. 1999, A\&A, 347, 640

Berné, O., Joblin, C., Deville, Y., et al. 2007, A\&A, 469, 575

Boersma, C., Bregman, J., \& Allamandola, L. J. 2014, ApJ, 795, 110

Boulanger, F., Falgarone, E., Puget, J. L., \& Helou, G. 1990, ApJ, 364, 136

Boutéraon, T., Habart, E., Ysard, N., et al. 2019, A\&A, 623, A135

Bowler, B. P., Waller, W. H., Megeath, S. T., Patten, B. M., \& Tamura, M. 2009, AJ, 137, 3685

Bron, E., Le Bourlot, J., \& Le Petit, F. 2014, A\&A, 569, A100

Bron, E., Agúndez, M., Goicoechea, J. R., \& Cernicharo, J. 2018, ArXiv e-prints, unpublished [arXiv:1801.01547]

Burke, J. R., \& Hollenbach, D. J. 1983, ApJ, 265, 223

Campeggio, L., Strafella, F., Maiolo, B., Elia, D., \& Aiello, S. 2007, ApJ, 668, 316

Cardelli, J. A., \& Clayton, G. C. 1991, AJ, 101, 1021

Cardelli, J. A., Clayton, G. C., \& Mathis, J. S. 1989, ApJ, 345, 245

Compiègne, M., Abergel, A., Verstraete, L., et al. 2007, A\&A, 471, 205

Compiègne, M., Abergel, A., Verstraete, L., \& Habart, E. 2008, A\&A, 491, 797

Compiègne, M., Verstraete, L., Jones, A., et al. 2011, A\&A, 525, A103

de Boer, K. S. 1983, A\&A, 125, 258

Demyk, K., Meny, C., Lu, X.-H., et al. 2017, A\&A, 600, A123

Desert, F.-X., Boulanger, F., \& Puget, J. L. 1990, A\&A, 237, 215

Draine, B. 2003, ARA\&A, 41, 241

Draine, B. T., \& Lee, H. M. 1984, ApJ, 285, 89

Duley, W. W., Zaidi, A., Wesolowski, M. J., \& Kuzmin, S. 2015, MNRAS, 447, 1242

Engelbracht, C., Blaylock, M., Su, K., et al. 2007, PASP, 119, 994

Fitzpatrick, E. L., \& Massa, D. 1986, ApJ, 307, 286

Flagey, N., Noriega-Crespo, A., Boulanger, F., et al. 2009, ApJ, 701, 1450

Gerin, M., Goicoechea, J. R., Pety, J., \& Hily-Blant, P. 2009, A\&A, 494, 977

Goicoechea, J. R., Pety, J., Gerin, M., et al. 2006, A\&A, 456, 565
Goicoechea, J. R., Pety, J., Gerin, M., Hily-Blant, P., \& Le Bourlot, J. 2009, A\&A, 498, 771

Gordon, K., Engelbracht, C., Fadda, D., et al. 2007, PASP, 119, 1019

Gordon, K. D., Baes, M., Bianchi, S., et al. 2017, A\&A, 603, A114

Gratier, P., Pety, J., Guzmán, V., et al. 2013, A\&A, 557, A101

Guzmán, V., Pety, J., Goicoechea, J. R., Gerin, M., \& Roueff, E. 2011, A\&A, 534, A49

Guzmán, V., Pety, J., Gratier, P., et al. 2012, A\&A, 543, L1

Guzmán, V. V., Goicoechea, J. R., Pety, J., et al. 2013, A\&A, 560, A73

Habart, E., Abergel, A., Walmsley, C. M., Teyssier, D., \& Pety, J. 2005, A\&A, 437, 177

Hily-Blant, P., Teyssier, D., Philipp, S., \& Güsten, R. 2005, A\&A, 440, 909

Hollenbach, D., \& Salpeter, E. E. 1971, ApJ, 163, 155

Hollenbach, D. J., \& Tielens, A. G. G. M. 1997, ARA\&A, 35, 179

Hollenbach, D. J., \& Tielens, A. G. G. M. 1999, Rev. Mod. Phys., 71, 173

Jones, A. P. 2012a, A\&A, 540, A1

Jones, A. P. 2012b, A\&A, 540, A2

Jones, A. P. 2012c, A\&A, 542, A98

Jones, A. P., \& Habart, E. 2015, A\&A, 581, A92

Jones, A. P., Duley, W. W., \& Williams, D. A. 1990, QJRAS, 31, 567

Jones, A. P., Fanciullo, L., Köhler, M., et al. 2013, A\&A, 558, A62

Jones, A. P., Ysard, N., Koehler, M., et al. 2014, Faraday Discuss., 168, 313

Jones, A. P., Köhler, M., Ysard, N., Bocchio, M., \& Verstraete, L. 2017, A\&A, 602, A46

Juvela, M. 2019, A\&A, 622, A79

Juvela, M., Ristorcelli, I., Pelkonen, V.-M., et al. 2011, A\&A, 527, A111

Juvela, M., Ristorcelli, I., Marshall, D. J., et al. 2015, A\&A, 584, A93

Juvela, M., Guillet, V., Liu, T., et al. 2018a, A\&A, 620, A26

Juvela, M., Malinen, J., Montillaud, J., et al. 2018b, A\&A, 614, A83

Juvela, M., Padoan, P., Ristorcelli, I., \& Pelkonen, V.-M. 2019, A\&A, 629, A63

Kim, S.-H., Martin, P. G., \& Hendry, P. D. 1994, ApJ, 422, 164

Köhler, M., Jones, A., \& Ysard, N. 2014, A\&A, 565, L9

Köhler, M., Ysard, N., \& Jones, A. P. 2015, A\&A, 579, A15

Kramer, C., Stutzki, J., \& Winnewisser, G. 1996, A\&A, 307, 915

Lada, E. A., Bally, J., \& Stark, A. A. 1991, ApJ, 368, 432

Laureijs, R. J., Clark, F. O., \& Prusti, T. 1991, ApJ, 372, 185

Le Gal, R., Herbst, E., Dufour, G., et al. 2017, A\&A, 605, A88

Le Petit, F., Nehmé, C., Le Bourlot, J., \& Roueff, E. 2006, ApJS, 164, 506

Li, A., \& Draine, B. T. 2001, ApJ, 550, L213

Li, A., \& Greenberg, J. M. 1997, A\&A, 323, 566

Martin, P. G., Roy, A., Bontemps, S., et al. 2012, ApJ, 751, 28

Mathis, J. S., \& Whiffen, G. 1989, ApJ, 341, 808

Mathis, J. S., Rumpl, W., \& Nordsieck, K. H. 1977, ApJ, 217, 425

Milman, A. S., Knapp, G. R., Knapp, S. L., \& Wilson, W. J. 1975, AJ, 80, 93

Neckel, T., \& Sarcander, M. 1985, A\&A, 147, L1

Ormel, C. W., Min, M., Tielens, A. G. G. M., Dominik, C., \& Paszun, D. 2011, A\&A, 532, A43

Ossenkopf, V., \& Henning, T. 1994, A\&A, 291, 943

Pety, J., Teyssier, D., Fossé, D., et al. 2005, A\&A, 435, 885

Pety, J., Goicoechea, J. R., Hily-Blant, P., Gerin, M., \& Teyssier, D. 2007, A\&A, 464, L41

Pety, J., Gratier, P., Guzmán, V., et al. 2012, A\&A, 548, A68

Philipp, S. D., Lis, D. C., Güsten, R., et al. 2006, A\&A, 454, 213

Pilleri, P., Montillaud, J., Berné, O., \& Joblin, C. 2012, A\&A, 542, A69

Pilleri, P., Reisenfeld, D. B., Zurbuchen, T. H., et al. 2015, ApJ, 812, 1

Planck Collaboration XXIV. 2011, A\&A, 536, A24

Pound, M. W., Reipurth, B., \& Bally, J. 2003, AJ, 125, 2108

Reach, W., Megeath, S., Cohen, M., et al. 2005, PASP, 117, 978

Roy, A., Martin, P. G., Polychroni, D., et al. 2013, ApJ, 763, 55

Schaerer, D., \& de Koter, A. 1997, A\&A, 322, 598

Siebenmorgen, R., \& Kruegel, E. 1992, A\&A, 259, 614

Smith, F. W. 1984, J. Appl. Phys., 55, 764

Stansberry, J., Gordon, K., Bhattacharya, B., et al. 2007, PASP, 119, 1038

Stepnik, B., Abergel, A., Bernard, J.-P., et al. 2003, A\&A, 398, 551

Swinyard, B. M., Ade, P., Baluteau, J.-P., et al. 2010, A\&A, 518, L4

Teyssier, D., Fossé, D., Gerin, M., et al. 2004, A\&A, 417, 135

Van De Putte, D., Gordon, K. D., Roman-Duval, J., et al. 2019, ApJ, 888, 22

Wakelam, V., Bron, E., Cazaux, S., et al. 2017, Mol. Astrophys., 9, 1

Warren, Jr., W. H., \& Hesser, J. E. 1977, ApJS, 34, 115

Weingartner, J. C., \& Draine, B. T. 2001a, ApJS, 134, 263

Weingartner, J. C., \& Draine, B. T. 2001b, ApJ, 548, 296

Ysard, N., Abergel, A., Ristorcelli, I., et al. 2013, A\&A, 559, A133

Ysard, N., Köhler, M., Jones, A., et al. 2015, A\&A, 577, A110

Ysard, N., Köhler, M., Jones, A., et al. 2016, A\&A, 588, A44

Zhou, S., Jaffe, D. T., Howe, J. E., et al. 1993, ApJ, 419, 190

Zubko, V., Dwek, E., \& Arendt, R. G. 2004, ApJS, 152, 211 


\section{Appendix A: Size distribution}

Size distributions of dust in THEMIS follow either a power law with an exponential cut-off, defined as

$$
\frac{\mathrm{d} n}{\mathrm{~d} a} \propto \begin{cases}a^{\alpha} & \text { if } \quad a<a_{\mathrm{t}} \\ a^{\alpha} \times \exp \left(-\left(\frac{a-a_{\mathrm{t}}}{a_{\mathrm{c}}}\right)^{3}\right) & \text { if } a \geq a_{\mathrm{t}}\end{cases}
$$

or a log-normal law, defined as

$$
\frac{\mathrm{d} n}{\mathrm{~d} a} \propto \frac{1}{a} \times \exp \left(-\left(\frac{\log \left(a / a_{0}\right)}{\sigma}\right)^{2}\right)
$$

where all the parameters for each dust distribution are listed in Table A.1.
Table A.1. Size distribution parameters for each dust population (see

\begin{tabular}{|c|c|c|c|c|c|c|c|}
\hline Name & Size & $\alpha$ & $a_{\min }$ & $a_{\max }$ & $a_{\mathrm{c}}$ & $a_{\mathrm{t}}$ & $a_{0}$ \\
\hline & & & {$[\mathrm{nm}]$} & {$[\mathrm{nm}]$} & {$[\mathrm{nm}]$} & {$[\mathrm{nm}]$} & {$[\mathrm{nm}]$} \\
\hline \multicolumn{8}{|c|}{ Core Mantle grains (CM) - (Diffuse ISM-like dust) } \\
\hline $\mathrm{a}-\mathrm{C}$ & p-law & 5 & 0.4 & 4900 & 10 & 50 & - \\
\hline $\mathrm{a}-\mathrm{C}: \mathrm{H} / \mathrm{a}-\mathrm{C}$ & $\log -n$ & - & 0.5 & 4900 & - & - & 7 \\
\hline a-Sil/a-C & $\log -n$ & - & 1 & 4900 & - & - & 8 \\
\hline \multicolumn{8}{|c|}{ Aggregated Mantle Mantle grains (AMM) } \\
\hline AMM & $\log -\mathrm{n}$ & - & 47.9 & 700 & - & - & 479 \\
\hline \multicolumn{8}{|c|}{ Aggregated Mantle Mantle Ice grains (AMMI) } \\
\hline AMMI & $\log -n$ & - & 91.2 & 700 & - & - & 610 \\
\hline
\end{tabular}
Appendix A for the equations).

Notes. p-law is a power law with an exponential tail, and log-n is a log-normal distribution. 
Appendix B: The Horsehead seen with Spitzer and Herschel
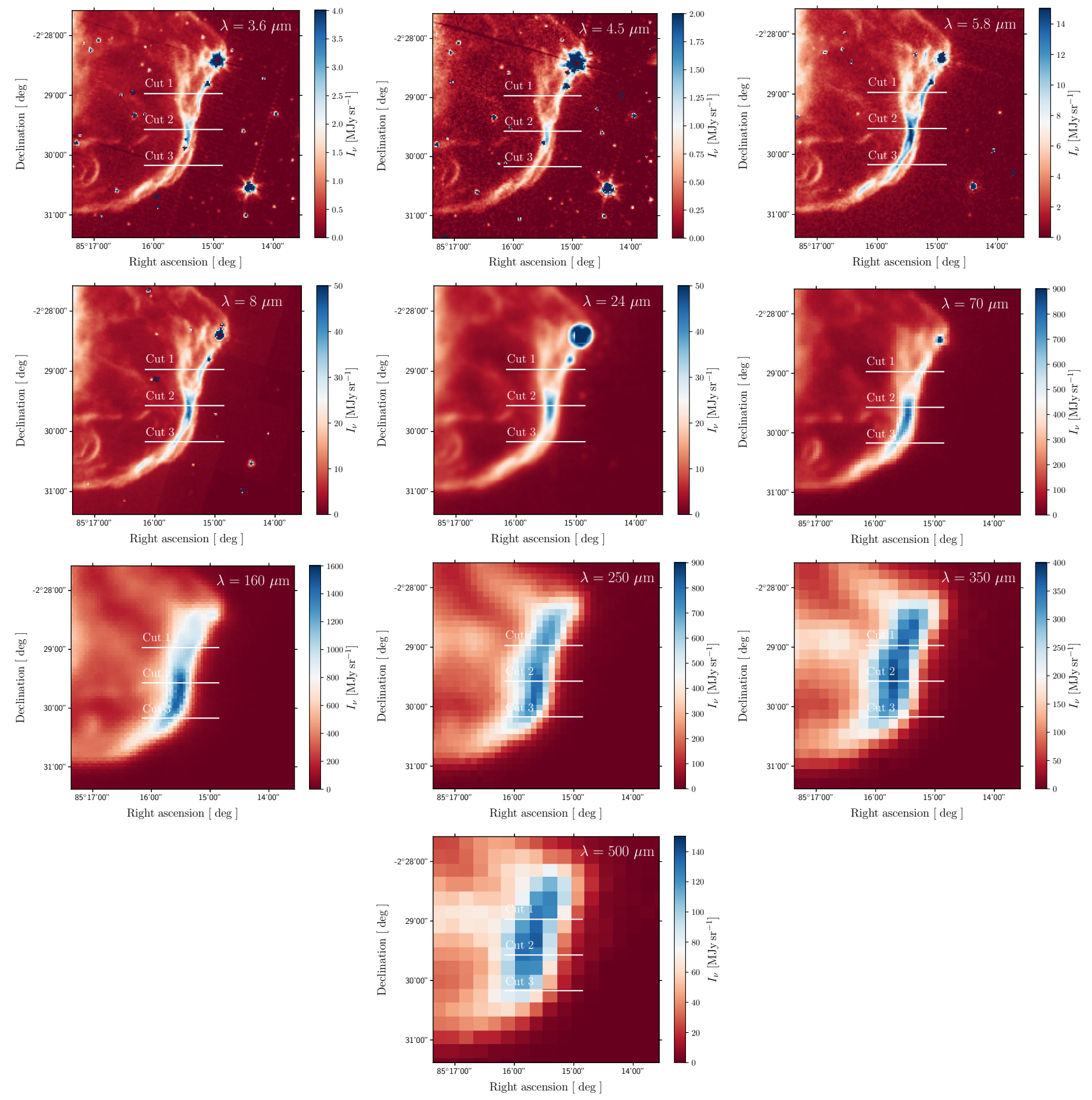

Fig. B.1. Horsehead seen in the ten photometric bands. The three solid white lines correspond to the three cuts we used. 